\title{
Novel one-shot inner bounds for unassisted fully quantum channels via rate splitting
}

\author{
Sayantan Charaborty* $\quad$ Aditya Nema* $\quad$ Pranab Sen*
}

\begin{abstract}
We prove the first non-trivial one-shot inner bounds for sending quantum information over an entanglement unassisted two-sender quantum multiple access channel (QMAC) and an unassisted two-sender two-receiver quantum interference channel (QIC). Previous works only studied the unassisted QMAC in the limit of many independent and identical uses of the channel also known as the asymptotic iid limit, and did not study the unassisted QIC at all. We employ two techniques, rate splitting and successive cancellation, in order to obtain our inner bound. Rate splitting was earlier used to obtain inner bounds, avoiding time sharing, for classical channels in the asymptotic iid setting. Our main technical contribution is to extend rate splitting from the classical asymptotic iid setting to the quantum one-shot setting. In the asymptotic iid limit our one-shot inner bound for QMAC approaches the rate region of Yard et al. [YDH05]. For the QIC we get novel non-trivial rate regions in the asymptotic iid setting. All our results also extend to the case where limited entanglement assistance is provided, in both one-shot and asymptotic iid settings. The limited entanglement results for one-setting for both QMAC and QIC are new. For the QIC the limited entanglement results are new even in the asymptotic iid setting.
\end{abstract}

\section{Introduction}

The multiple access channel (MAC), where two independent senders Alice (A) and Bob (B) have to send their respective messages to a single receiver Charlie $(C)$ via a communication channel with two inputs and one output, is arguably the simplest multiterminal channel. Yet, it abstracts out important practical situations like several independent users transmitting their respective messages to a base station. Ahlswede [Ahl71], and independently Liao [Lia72], obtained the first optimal rate region for the classical MAC in the asymptotic iid setting, using a powerful method called simultaneous decoding. Their region looks like the one in Figure 1, where $I(A: B):=H(A)+H(B)-H(A B)$ denotes the mutual information between two jointly distributed random variables $A, B$. Simultaneous decoding means that Charlie is able to decode any point in the rate region, e.g. point $\mathrm{P}$ in Figure 1, by a one-step procedure. Later on, other authors obtained the same rate region in a computationally less intensive fashion by using successive cancellation and time sharing. In successive cancellation decoding Charlie first decodes Alice's message and then uses it as an additional channel output in order to next decode Bob's message, or vice versa. In other words, Charlie can either decide to decode point $S$ or point $T$ in Figure 1, In order

${ }^{*}$ School of Technology and System Science, Tata Institute of Fundamental Research, Mumbai, India. Email: \{kingsbandz, aditya.nema30, pranab.sen.73\}@gmail.com 


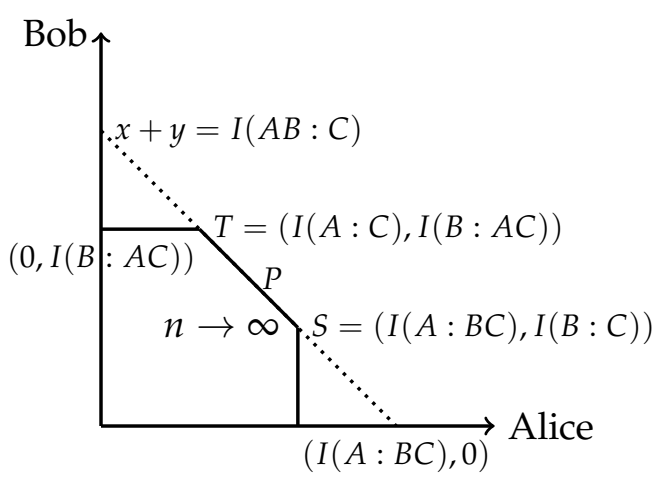

Figure 1: Achievable rate region per channel use for the classical MAC in the asymptotic iid setting.

to decode another point in the rate region, e.g. point $\mathrm{P}$ in Figure 1, Charlie first figures out the convex combination $(\alpha, 1-\alpha)$ of points $S$ and $T$ that would give point $P$. Out of $n$ iid channel uses, Charlie then decodes the first $\alpha n$ uses according to point $S^{\prime}$ 's decoding strategy and the remaining $(1-\alpha) n$ channel uses according to point $\mathrm{T}^{\prime}$ 's decoding strategy. This idea is called time sharing.

The interference channel is another important channel where sender Alice wants to send her message to receiver Charlie and sender Bob, whose message is independent of that of Alice, wants to send his message to receiver Damru via a communication channel with two inputs and two outputs. It abstracts out the important practical situation where independent sender-receiver pairs are communicating simultaneously via a noisy medium. Han and Kobayashi [HK81] (see also [CMGE08]) obtained the best known inner bound for this channel in the classical asymptotic iid setting.

The multiple access and interference channels can be defined in the context of quantum information theory also. Early work studied the sending of classical information over a quantum MAC, without [Win01] or with [HDW08] entanglement assistance, in the asymptotic iid setting. These works obtained the natural quantum analogues of the optimal classical rate regions using successive cancellation and time sharing. Later, Fawzi et al. [FHS ${ }^{+} 12$ ] and Sen [Sen12] studied the sending of classical information over a quantum interference channel in the asymptotic iid setting by first obtaining a simultaneous decoder for the quantum MAC. The latter paper managed to obtain the natural quantum analogue of the Han-Kobayashi inner bound.

For a variety of reasons recent research in Shannon theory has studied in depth the one-shot setting where the channel can be used only once. This is the most general setting and subsumes the asymptotic iid, asymptotic non-iid aka information spectrum, and finite block length settings. Ideally, the one-shot inner bounds should match or supersede the best inner bounds for the respective channels in the asymptotic iid setting. Sen [Sen18a] obtained the natural one-shot quantum analogues of best known classical rate regions for sending classical information over entanglement unassisted and assisted quantum MACs and quantum interference channels. His one-shot inner bounds, obtained by simultaneous decoding, approach the optimal inner bounds known earlier for the classical and quantum asymptotic iid settings.

Note that presence of shared randomness does not affect the rates of sending classical or quantum information over channels. Also the rates of sending quantum information and classical information over entanglement assisted quantum channels are related by a factor of two because of quantum teleportation. So the main setting left unstudied in the above works is the 
setting of sending quantum information over an entanglement unassisted quantum channel i.e. the senders and the receivers do not share any entanglement prior to the beginning of the protocol. The first works to address this setting looked at a point-to-point quantum channel in the asymptotic iid setting [Llo97,Sho02], culminating in the work of Devetak [Dev05] which showed with full rigour that the regularised coherent information from sender $A$ to receiver $B$ defined by $I^{*}(A>B):=\lim _{k \rightarrow \infty} I\left(A^{k}>B^{k}\right) / k, I\left(A^{k}>B^{k}\right):=H\left(B^{k}\right)-H\left(A^{k} B^{k}\right)$ where $A^{k} B^{k}$ is defined by the channel action $\left(\mathcal{N}^{A^{\prime} \rightarrow B}\right)^{\otimes k}$ on an arbitrary (in general entangled) pure state $|\sigma\rangle^{A^{k}\left(A^{\prime}\right)^{k}}$, is the capacity of an unassisted quantum channel in the asymptotic iid limit. Hayden et al. [HHYW07]. showed that one can recover Devetak's result using a technique called decoupling

These works naturally lead one to consider unassisted multiterminal quantum channels. To the best of our knowledge, the only inner bound known for the unassisted QIC is what one would obtain by treating the channel as two independent unassisted point to point channels. For the unassisted QMAC more is known. Yard et al. [YDH05] showed that the natural quantum analogue of the classical rate region, with mutual information replaced by regularised coherent information as in Figure 2, is an inner bound for the unassisted quantum MAC (QMAC) in the asymptotic iid setting. They proved their inner bound by time sharing and a suitable adaptation of successive cancellation.

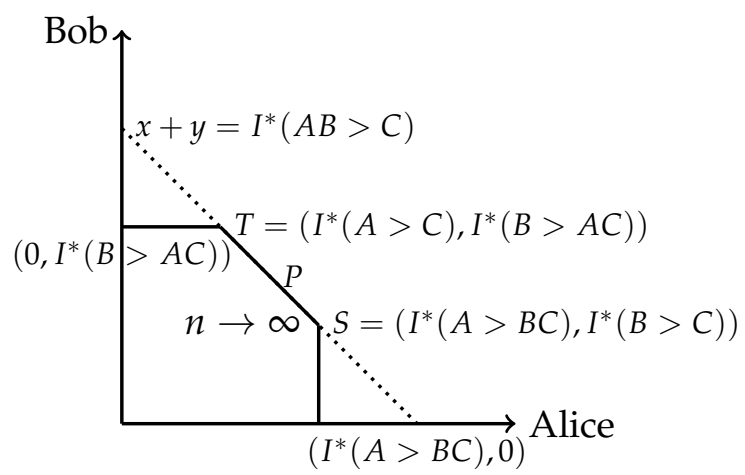

Figure 2: Achievable rate region for the unassisted quantum MAC per channel use in the asymptotic iid setting.

The above works behoove one to consider the problem of sending quantum information over an unassisted quantum channel in the one-shot setting. Buscemi and Datta [BD10] proved the first one-shot achievability result for the unassisted point-to-point channel in terms of smooth modified Rényi entropies. Their result was generalised by Dupuis [Dup10] to the case where the receiver has some side information about the sender's message. In the asymptotic iid limit, these one-shot results approach the regularised coherent information obtained in earlier works.

It is thus natural to study inner bounds for the unassisted QMAC in the one-shot setting. In this paper we take the first steps towards this problem. Observe that successive cancellation can only give the two endpoints $S$ and $T$ of the dominant line of the pentagonal rate region in Figure 2 . Since time sharing cannot be used in the one-shot setting, it is not clear how to obtain other rate tuples like the point $P$. An alternative would be to develop a simultaneous decoder for the QMAC which can obtain a point like $P$ directly, but that is a major open problem with connections to the notorious simultaneous smoothing open problem [CNS21].

Instead in this paper, we take inspiration from another powerful classical channel coding technique called rate splitting. Grant, et al. [GRUW01] showed that it is possible to 'split' Alice into two 
senders Alice $_{0}$ and Alice ${ }_{1}$, each sending disjoint parts of Alice's original message, such that any point in the pentagonal rate region of Figure 2 like $\mathrm{P}$ can be obtained without time sharing by a successive cancellation process where Charlie first decodes Alice ${ }_{0}$ 's message, then Bob's message using Alice $_{0}$ 's message as side information and finally Alice ${ }_{1}$ 's message using Bob's and Alice ${ }_{0}$ 's messages as side information. Though Grant et al.'s rate splitting technique was developed for the classical MAC in the asymptotic iid setting, in this paper we show how it can be adapted to the oneshot quantum setting. This is a non-trivial task, which we tackle in two steps. In the first step we use ideas from Yard et al. [YDH05] and Dupuis [Dup10] and suitably adapt successive cancellation to the one-shot unassisted quantum setting. In the second step, we adapt the rate splitting function of Grant et al. [GRUW01] to the one-shot quantum setting. Our one-shot rates are in terms of the smooth coherent Rényi-2 information defined in Section[2. Since the smooth coherent Rényi-2 information is not known to possess a chain rule with equality, we get an achievable rate region of the form in Figure 3. Our achievable rate region is a subset of the 'ideal' pentagonal rate region shown by the dashed line. Nevertheless, using a quantum asymptotic equipartition result of Tomamichel et al. [TCR09], we show that this 'subpentagonal' achievable rate region approaches the 'pentagonal' region of Yard et al. [YDH05] (equal to the region demarcated by the dashed line) in the iid limit. The reason why splitting of Alice into Alice ${ }_{0}$ and Alice ${ }_{1}$ allows one to obtain a $\left(0, I_{2}^{\sqrt{\epsilon}}(A B>C)\right)$ (

Figure 3: One-shot achievable rate region for the unassisted QMAC (for single channel use only), contained inside the 'ideal' pentagonal region demarcated by the dashed line, and approaching it in the asymptotic iid limit. $O(\log \epsilon)$ additive factors have been ignored in the figure.

'middle' rate point like $P$, in addition to the 'corner' points $S$ and $T$, is as follows. The rate point $P$ is the projection onto the (Alice, Bob) plane of the 'corner' rate point $P^{\prime}$ in the (Alice ${ }_{0}$, Bob, Alice 1 ) space where the rates of Alice ${ }_{0}$ and Alice ${ }_{1}$ are summed to obtain Alice's rate. The point $P^{\prime}$ can be obtained by a 3-step successive cancellation decoding. Note that the split of Alice depends on the rate point $P$ to be attained.

We now state our result for the unassisted QIC. The trivial inner bound treats the QIC as two independent unassisted point to point channels from Alice to Charlie and Bob to Damru. Rate splitting and successive cancellation can be similarly used to obtain non-trivial rate regions for the unassisted QIC where one party, say Alice, sacrifices her rate in order to boost Bob's rate with respect to the trivial inner bound. The situation is summarised in Figure 5. Though the discussion above only involved unassisted QMAC and QIC, our actual results also hold for the QMAC and QIC with limited entanglement assistance. However they seem to be inferior to the known results when entanglement assistance is unlimited [Sen18b]. 


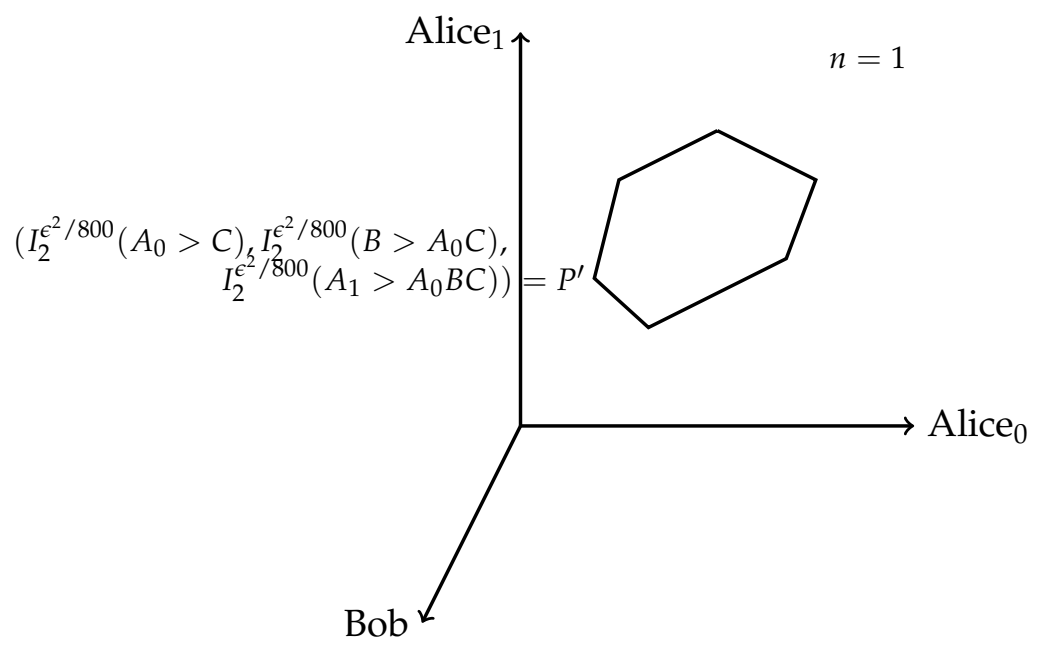

Figure 4: The 'corner' point $P^{\prime}$ can be obtained by successive cancellation following the order Alice $_{0} \rightarrow$ Bob $\rightarrow$ Alice $_{1}$ with splitting of Alice followed by one use of the unassisted QMAC. Point $P^{\prime}$ projects down to point $P$ in Figure 3, Only the 'dominant face' of the rate region is shown. Successive cancellation can only obtain the corner points of the dominant face and all 'sub-points' by 'resource wasting'. It cannot obtain 'middle' points of the 'dominant' face. $O(\log \epsilon)$ additive factors have been ignored in the figure.

\section{Preliminaries}

\subsection{Notation}

We will use the following conventions throughout the rest of the paper :

1. Suppose that $|\omega(U)\rangle^{X A^{\prime} B^{\prime}}$ be a generic intermediary state (defined in Section 2.6), where $X$ is a placeholder for other systems involve din the protocol. Suppose we are given a channel $\mathcal{N}^{A^{\prime} B^{\prime} \rightarrow C}$ and its corresponding Stinespring dilation $\mathcal{U}_{\mathcal{N}}^{A^{\prime} B^{\prime} \rightarrow C E}$. Then, we denote the state $\mathcal{U}_{\mathcal{N}}|\omega(U)\rangle^{X A^{\prime} B^{\prime}}$ by the symbol $|\omega(U)\rangle^{X C E}$. Although the two states are denoted using the same greek letter, we differentiate them by the systems on which they are defined. These systems will always be explicitly mentioned whenever we make use of this convention.

2. We will use the same rule for control states, For example, suppose $|\sigma\rangle^{A^{\prime \prime} A^{\prime} B^{\prime \prime} B^{\prime}}$ is a control state for some channel coding protocol. Suppose we are given the channel $\mathcal{N}^{A^{\prime} B^{\prime} \rightarrow C}$ Then we use the following convention

$$
\sigma^{A^{\prime \prime} B^{\prime \prime C}}:=\mathcal{N} \cdot \sigma^{A^{\prime \prime} A^{\prime} B^{\prime \prime} B^{\prime}}
$$

We will use this convention while specifying entropic quantities. It will be clear from context which state we refer to. For example, consider the expressions $H_{\min }^{\epsilon}\left(A^{\prime \prime}\right)_{\sigma}$ and $I_{\min }^{\epsilon}\left(A^{\prime \prime} \mid C\right)_{\sigma}$. It is clear from the arguments of the entropic expressions that in the first case $\sigma=\sigma^{A^{\prime \prime}} A^{\prime} B^{\prime \prime} B^{\prime}$ and in the second case $\sigma=\sigma^{A^{\prime \prime} B^{\prime} C}$.

3. We will, on several occasions use the operator op ${ }^{X \rightarrow Y A^{\prime} B^{\prime}}\left(|\omega(U)\rangle^{X Y A^{\prime} B^{\prime}}\right)$. To lessen the burden on notation, whenever we use this operator, we will not mention the systems on which the argument of the op operator is defined. It will however always mention the domain and range of the op operator in these cases to avoid any confusion. 


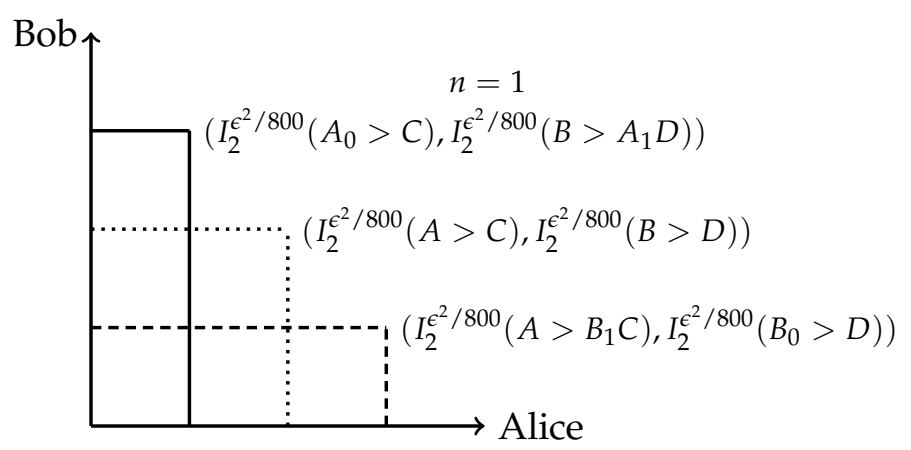

Figure 5: One-shot achievable rate region (for single channel use only) for the unassisted QIC. The trivial region is shown dotted. Alice can sacrifice her rate in order to boost Bob's rate with respect to the trivial region, as shown by the solid rectangle. The dashed rectangle can be similarly obtained by Bob sacrificing his rate in order to boost Alice's. $O(\log \epsilon)$ additive factors have been ignored in the figure.

\subsection{Smooth Entropies}

For a pair of subnormalised density matrices $\rho$ and $\sigma$ in the same Hilbert space their purified distance is denoted by $P(\rho, \sigma):=\sqrt{1-F(\rho, \sigma)^{2}}$ where $F(\rho, \sigma):=\|\sqrt{\rho} \sqrt{\sigma}\|_{1}+\sqrt{(1-\operatorname{Tr}[\rho]) \cdot(1-\operatorname{Tr}[\sigma])}$ is the generalised fidelity and $\|\cdot\|_{1}$ is the Schatten 1-norm. We use $\sigma \approx_{\epsilon} \rho$ as a shorthand for $P(\sigma, \rho) \leq \epsilon$.

The Shannon (aka von Neumann) entropy for a normalised quantum state $\rho^{A}$ is defined by $H(A)_{\rho}:=-\operatorname{Tr}[\rho \log \rho]$. For a bipartite quantum state $\rho^{A B}$, the $\epsilon$-smooth sandwiched Rényi-2 coherent information is defined as $I_{2}^{\epsilon}(A>B)_{\rho}:=H_{2}^{\epsilon}(A \mid E)_{\rho}$ where $|\rho\rangle^{A B E}$ is a purification of $\rho^{A B}$. Above, the $\epsilon$-smooth sandwiched Rényi-2 conditional entropy is defined as

$$
\begin{aligned}
& H_{2}^{\epsilon}(A \mid E)_{\rho}:= \\
& -2 \log \min _{\left(\rho^{\prime}\right)^{A E} \approx_{\epsilon} \rho^{A E}} \min _{\sigma^{E}}\left\|\left(\mathbb{1}^{A} \otimes\left(\sigma^{E}\right)^{-1 / 4}\right) \cdot\left(\rho^{\prime}\right)^{A E}\right\|_{2},
\end{aligned}
$$

where $\sigma^{E}$ ranges over non-singular normalised states over $E,\|\cdot\|_{2}$ is the Schatten 2-norm aka Frobenius norm and $M \cdot N:=M N M^{\dagger}$ for operators $M, N$ in the same Hilbert space. The $\epsilon$-smooth sandwiched Rényi-2 coherent information is now defined by $I_{2}^{\epsilon}(A>B)_{\rho}:=H_{2}^{\epsilon}(A \mid E)_{\rho}$, where $|\rho\rangle^{A B E}$ is a purification of $\rho^{A B}$.

The $\epsilon$-smooth conditional min-entropy is given by

$$
\begin{aligned}
& H_{\min }^{\epsilon}(A \mid E)_{\rho}:= \\
& -\log \min _{\left(\rho^{\prime}\right)^{A E} \approx_{\epsilon} \rho^{A E}} \min _{\sigma^{E}:\left(\rho^{\prime}\right)^{A E} \leq \mathbb{1}^{A} \otimes \sigma^{E}} \operatorname{Tr}\left[\sigma^{E}\right],
\end{aligned}
$$

where $\sigma^{E}$ ranges over positive semidefinite operators on $E$. Then the $\epsilon$-smooth coherent min-information aka the negative of the $\epsilon$-smooth conditional max-entropy is given by

$$
I_{\min }^{\epsilon}(A>B)_{\rho}:=-H_{\max }^{\epsilon}(A \mid B)_{\rho}:=H_{\min }^{\epsilon}(A \mid E)_{\rho},
$$

where again $|\rho\rangle^{A B E}$ is a purification of $\rho^{A B}$. The unconditional smooth entropies are now defined from the conditional ones by taking the conditioning system to be one dimensional. 
As shown in [Dup10], the smooth sandwiched Rényi-2 conditional entropy upper bounds the smooth conditional min-entropy. The smooth conditional min-entropy is further lower bounded by the familiar conditional Shannon entropy in the amortised sense in the asymptotic iid limit [TCR09], a result that is sometimes referred to as the fully quantum asymptotic equipartition property. To summarise, the smooth sandwiched Rényi-2 coherent information upper bounds the Shannon coherent information in the amortised sense in the asymptotic iid limit.

We will now state some properties on the smooth conditional min entropy that we will use throughout the rest of the paper.

Fact 2.1 (Chaining for Smooth min-entropy [VDTR13, DBWR14]) Let $\epsilon>0$ and $\epsilon^{\prime}, \epsilon^{\prime \prime} \geq 0$ and let $\rho^{A B C}$ be a quantum state. Then

$$
H_{\min }^{\epsilon+2 \epsilon^{\prime}+\epsilon^{\prime \prime}}(A B \mid C)_{\rho} \geq H_{\min }^{\epsilon^{\prime}}(A \mid B C)_{\rho}+H_{\min }^{\epsilon^{\prime \prime}}(B \mid C)_{\rho}-\log \frac{2}{\epsilon^{2}}
$$

Fact 2.2 (Unitary Invariance of Smooth min-entropy) Given $\epsilon \geq 0$, a quantum state $\rho^{A B}$ and isometries $U: \mathcal{H}_{A} \rightarrow \mathcal{H}_{C}$ and $V: \mathcal{H}_{B} \rightarrow \mathcal{H}_{D}$, define the state $\sigma^{C D}:=(U \otimes V) \rho^{A B}\left(U^{\dagger} \otimes V^{\dagger}\right)$. Then

$$
H_{\min }^{\epsilon}(A \mid B)_{\rho}=H_{\min }^{\epsilon}(C \mid D)_{\sigma}
$$

Fact 2.3 (Continuity of Smooth min-entropy) Given two quantum states $\rho^{A B}$ and $\sigma^{A B}$ such that $P\left(\rho^{A B}, \sigma^{A B}\right) \leq$ $\delta$ and $\epsilon>0$, then

$$
\left|H_{\min }^{\epsilon}(A \mid B)_{\rho}-H_{\min }^{\epsilon}(A \mid B)_{\sigma}\right| \leq c \cdot \delta^{\prime}
$$

where $c$ is an absolute constant and depends on the dimensions of system $A$ and $B$ and $\delta^{\prime}=\sqrt{\delta^{2}+2 \epsilon \delta}$

The proofs of both Theorem 2.2 and Theorem 2.3 can be found in [TCR10].

Fact 2.4 (Quantum Asymptotic Equipartition Property [TCR09]) Given a bipartite quantum state $\rho^{A B}$ on the system $\mathcal{H}_{A} \otimes \mathcal{H}_{B}, \epsilon>0$, an integer $n \in \mathbb{N}$ and the iid extension of the state $\rho_{A B}^{n}$ it holds that

$$
\lim _{\epsilon \rightarrow 0} \lim _{n \rightarrow \infty} \frac{1}{n} H_{\min }^{\epsilon}\left(A^{n} \mid B^{n}\right)_{\rho^{n}}=H(A \mid B)_{\rho}
$$

\subsection{The op Operator}

One of the main technical tools we use in this paper, which is a workhorse in most of our proofs, is the notion of mapping a vector into an operator. This operation is denoted simply by 'op' and we compile some of its properties in this section for completeness. The interested reader is referred to [Dup10] for further details.

Definition 2.5 ('The op operator') Given the systems $A$ and $B$, fix the standard bases $\left|a_{i}\right\rangle^{A}$ and $\left|b_{j}\right\rangle^{B}$. Then we define op ${ }^{A \rightarrow B}: A \otimes B \rightarrow L(A, B)$ as

$$
\text { op }^{A \rightarrow B}\left(\left|a_{i}\right\rangle\left|b_{j}\right\rangle\right):=\left|b_{j}\right\rangle\left\langle a_{i}\right| \quad \forall i, j
$$

Notice that this definition is basis dependant and hence whenever we use this operator a choice of bases is implied, although not always explicitly mentioned. 
Fact 2.6 Let $|\psi\rangle^{A B}$ and $|\varphi\rangle^{A C}$ be vectors on the systems $A B$ and $A C$ respectively. Then

$$
\text { op }^{A \rightarrow C}\left(|\varphi\rangle^{A C}\right)|\psi\rangle^{A B}=\text { op }^{A \rightarrow B}\left(|\psi\rangle^{A B}\right)|\varphi\rangle^{A C}
$$

Fact 2.7 Given a vector $|\psi\rangle^{A B}$, let $|\Phi\rangle^{A A^{\prime}}$ be an EPR state, where $A \cong A^{\prime}$. Then,

$$
\sqrt{|A|} \text { op }^{A \rightarrow B}\left(|\psi\rangle^{A B}\right)|\Psi\rangle^{A A^{\prime}}=|\psi\rangle^{A^{\prime} B}
$$

Fact 2.8 For all vectors $|\psi\rangle^{A B}$ and any $M^{A \rightarrow C}$,

$$
\text { op }^{C \rightarrow B}(M|\psi\rangle)=\text { op }^{A \rightarrow B}(|\psi\rangle) M^{T}
$$

Fact 2.9 For all $|\psi\rangle^{A B}$,

$$
\operatorname{Tr}_{B}\left[\psi^{A B}\right]=\mathrm{op}^{B \rightarrow A}(|\psi\rangle) \mathrm{op}^{B \rightarrow A}(|\psi\rangle)^{\dagger}
$$

\subsection{The Smooth Single Sender Decoupling Theorem}

Fact 2.10 Decoupling Theorem Given $\epsilon>0$ a density matrix $\rho^{A E}$ and any completely positive operator $\mathcal{T}^{A \rightarrow R}$, define $\omega^{A^{\prime} R}:=\left(\mathcal{T} \otimes \mathbb{I}^{A^{\prime}}\right) \Phi^{A A^{\prime}}$. Then

$$
\int_{\mathbf{U}(\mathbf{A})}\left\|\mathcal{T}(U \cdot \rho)-\omega^{E} \otimes \rho^{R}\right\|_{1} \leq 2^{-\frac{1}{2} H_{2}^{\epsilon}\left(A^{\prime} \mid R\right)_{\omega}-\frac{1}{2} H_{2}^{\epsilon}(A \mid E)_{\rho}}+8 \epsilon
$$

where the integration is over the Haar measure on the set of all unitaries on the system $A$, denoted by $\mathbb{U}(\mathbb{A})$.

The single sender decoupling theorem implies the following channel coding theorem.

Fact 2.11 [Dup10, Theorem 3.14] Let $|\psi\rangle^{A B R}$ be a pure state, $\mathcal{N}^{A^{\prime} \rightarrow C}$ be any CPTP superoperator with Stinespring dilation $U_{\mathcal{N}}^{A^{\prime} \rightarrow C E}, N$ and complementary channel $\overline{\mathcal{N}}^{A^{\prime} \rightarrow E}$, let $\omega^{A^{\prime \prime} C E}:=U_{\mathcal{N}} \cdot \sigma^{A^{\prime \prime}} A^{\prime}$, where $\sigma^{A^{\prime \prime} A^{\prime}}$ is any pure state and $A^{\prime \prime} \cong A^{\prime}$, and let $\epsilon>0$. Then, there exists an encoding partial isometry $V^{A \rightarrow A^{\prime}}$ and a decoding superoperator $\mathcal{D}^{C B \rightarrow A B}$ such that:

$$
\left\|\overline{\mathcal{N}}\left(V \cdot \psi^{A R}\right)-\omega^{E} \otimes \psi^{R}\right\|_{1} \leq 2 \sqrt{2 \delta_{1}}+\delta_{2}
$$

and

$$
\left\|(\mathcal{D} \circ \mathcal{N} \circ \mathcal{E}) \psi^{A B R}-\psi^{A B R}\right\|_{1} \leq 2 \sqrt{\left(4 \sqrt{2 \delta_{1}}+2 \delta_{2}\right)}
$$

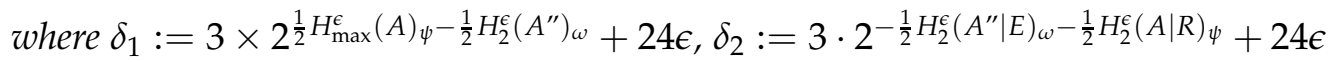

\subsection{Miscellaneous Useful Facts}

Fact 2.12 Given states $\rho^{A B C}, \sigma^{A}, \eta^{C}, \sigma^{A B}, \omega^{B C}$ such that

$$
\begin{aligned}
& \left\|\rho^{A B C}-\sigma^{A} \otimes \omega^{B C}\right\|_{1} \leq \epsilon_{1} \\
& \left\|\rho^{A B C}-\sigma^{A B} \otimes \eta^{C}\right\|_{1} \leq \epsilon_{2}
\end{aligned}
$$

it holds that

$$
\left\|\rho^{A B C}-\sigma^{A} \otimes \sigma^{B} \otimes \eta^{C}\right\|_{1} \leq 2 \epsilon_{1}+\epsilon_{2}
$$

Fact 2.13 For any two density matrices $\rho$ and $\sigma$ and any real $c \in \mathbb{R}$, the following holds true:

$$
\|\rho-\sigma\|_{1} \leq 2\|c \rho-\sigma\|_{1}
$$




\subsection{Almost CPTP Maps}

In this section we will precisely define the two main technical tools used in the paper: intermediary states and almost CPTP maps. We describe the objects in this section in the context of a QMAC $\mathcal{N} A^{\prime} B^{\prime} \rightarrow C$. We assume that we ar given the control state $|\sigma\rangle^{A^{\prime \prime} A^{\prime} B^{\prime \prime} B^{\prime}}=|\Omega\rangle^{A^{\prime \prime} A^{\prime}}|\Delta\rangle^{B^{\prime \prime} B^{\prime}}$ and the task is for the 2 senders Alice and Bob to transmit the $A$ and $B$ systems of the states $|\psi\rangle^{A R_{1}}$ and $|\varphi\rangle^{B R_{2}}$ via the channel after some suitable encoding. At the very outset we use the isometric embeddings $W_{1}^{A \rightarrow A^{\prime \prime}}$ and $W_{2}^{B \rightarrow B^{\prime \prime}}$ to map the systems $A$ and $B$ to $A^{\prime \prime}$ and $B^{\prime \prime}$. To ease notation, we use the symbols $|\psi\rangle^{A^{\prime \prime} R_{1}}$ and $|\varphi\rangle^{B^{\prime \prime} R_{2}}$ to denote the states $W_{1}|\psi\rangle$ and $W_{2}|\varphi\rangle$. First, suppose $U_{1}$ is a Haar random unitary and the $U_{2}$ is a random unitary sampled from the distribution $\mu$.

Definition 2.14 (Intermediary State) We define the intermediary state with respect to the unitary $U_{2}$ as

$$
\left|\omega\left(U_{2}\right)\right\rangle^{A^{\prime \prime} A^{\prime} B^{\prime} R_{2}}:=\sqrt{B^{\prime \prime}}\left(\mathrm{op}^{B^{\prime \prime} \rightarrow A^{\prime \prime} A^{\prime} B^{\prime}}(\sigma) U_{2}^{B^{\prime \prime}}|\varphi\rangle^{B^{\prime \prime} R_{2}}\right)
$$

We will need one more definition, that of the almost CPTP map.

Definition 2.15 (Almost CPTP) We define the linear map $\mathcal{T}^{B^{\prime \prime} \rightarrow R_{2}}$ as an almost CPTP if $\mathcal{T}$ has the following properties:

1. $\mathcal{T}$ is $C P$.

2. $\operatorname{Tr}\left[\mathcal{T}\left(\pi^{B^{\prime \prime}}\right)\right] \in[1-\delta, 1+\delta]$ for some small $\delta \geq 0$.

3. $\int \mathcal{T}\left(U_{2}^{B^{\prime \prime}} \cdot \xi\right) d \mu=\operatorname{Tr}[\xi] \mathcal{T}\left(\pi^{B^{\prime \prime}}\right)$

Lemma 2.16 When the measure $\mu$ is set to be the Haar measure on the unitary group on $B^{\prime \prime}$, there exists an almost $C P T P \mathcal{T}^{B^{\prime \prime} \rightarrow R_{2}}$ such that, with constant probability,

$$
H_{\min }^{\epsilon}\left(A^{\prime \prime} \mid R_{2}\right)_{\omega\left(U_{2}\right)} \geq H_{\min }^{\frac{\epsilon^{2}}{36}}\left(A^{\prime \prime} \mid B^{\prime \prime}\right)_{\sigma}-O(1)
$$

Proof:[Proof of Theorem 2.16] Let

$$
\mathcal{T}^{B^{\prime \prime} \rightarrow R_{2}}(\xi):=\left|B^{\prime \prime}\right|\left(\mathrm{op}^{B^{\prime \prime} \rightarrow R_{2}}(\varphi) \cdot \xi\right)
$$

Firstly, it is clear that $\mathcal{T}$ is CP. Next, we see that

$$
\begin{aligned}
\operatorname{Tr}\left[\mathcal{T}\left(\pi^{B^{\prime \prime}}\right)\right] & =\operatorname{Tr}\left[\mathrm{op}^{B^{\prime \prime} \rightarrow R_{2}}(\varphi) \mathrm{op}^{B^{\prime \prime} \rightarrow R_{2}}(\varphi)^{\dagger}\right] \\
& =\operatorname{Tr}\left[\operatorname{Tr}_{B^{\prime \prime}}(\varphi)\right] \\
& =1
\end{aligned}
$$

It is another easy verification, using the properties of Haar integrals, that

$$
\int \mathcal{T}\left(U_{1} \cdot \xi\right) d \mu=\operatorname{Tr}[\xi] \mathcal{T}\left(\pi^{B^{\prime \prime}}\right)
$$


This shows that $\mathcal{T}$ is indeed an almost CPTP. Again, using the properties of the op operator we see that

$$
\begin{aligned}
\mathcal{T}\left(\left(U_{2}^{B^{\prime \prime}}\right)^{T} \cdot \sigma\right) & =\left|B^{\prime \prime}\right|\left(\operatorname{op}^{B^{\prime \prime} \rightarrow R_{2}}(\varphi) \cdot\left(\left(U_{2}^{B^{\prime \prime}}\right)^{T} \cdot \sigma^{A^{\prime \prime} B^{\prime \prime} A^{\prime} B^{\prime}}\right)\right) \\
& =\left|B^{\prime \prime}\right|\left(\operatorname{op}^{B^{\prime \prime} \rightarrow R_{2}}\left(U_{2}^{B^{\prime \prime}} \varphi\right) \cdot \sigma^{A^{\prime \prime} B^{\prime \prime} A^{\prime} B^{\prime}}\right) \\
& =\left|B^{\prime \prime}\right|\left(\operatorname{op}^{B^{\prime \prime} \rightarrow A^{\prime \prime} A^{\prime} B^{\prime}}(\sigma) \cdot\left(U_{2}^{B^{\prime \prime}} \cdot \varphi^{B^{\prime \prime} R_{2}}\right)\right) \\
& =\omega\left(U_{2}\right)
\end{aligned}
$$

Now, suppose that $\tilde{\sigma}$ is the optimiser in the definition of $H_{\min }^{\epsilon}\left(A^{\prime \prime} \mid B^{\prime \prime}\right)_{\sigma}$ and that $\|\tilde{\sigma}-\sigma\|_{1} \leq 2 \epsilon$. Suppose also that $\lambda^{B^{\prime \prime}}$ be a positive semidefinite matrix such that $\operatorname{Tr}\left[\lambda^{B^{\prime \prime}}\right]=2^{-H_{\min }^{\epsilon}\left(A^{\prime \prime} \mid B^{\prime \prime}\right)_{\sigma}}$ and

$$
\tilde{\sigma}^{A^{\prime \prime} B^{\prime \prime}} \leq \mathbb{I}^{A^{\prime \prime}} \otimes \lambda^{B^{\prime \prime}}
$$

Then, using the fact that $\mathcal{T}$ is a CP map, we see that

$$
\begin{gathered}
\mathcal{T}\left(\left(U_{2}^{B^{\prime \prime}}\right)^{T} \cdot \tilde{\sigma}^{A^{\prime \prime} B^{\prime \prime}}\right) \leq \mathbb{I}^{A^{\prime \prime}} \otimes \mathcal{T}\left(\left(U_{2}^{B^{\prime \prime}}\right)^{T} \cdot \lambda^{B^{\prime \prime}}\right)^{R_{2}} \\
\Longrightarrow \omega\left(U_{2}\right)^{A^{\prime \prime} R_{2}} \leq \mathbb{I}^{A^{\prime \prime}} \otimes \mathcal{T}\left(\left(U_{2}^{B^{\prime \prime}}\right)^{T} \cdot \lambda^{B^{\prime \prime}}\right)^{R_{2}}
\end{gathered}
$$

First notice that, by properties 2 and 3 of almost CPTP maps,

$$
\begin{aligned}
\int \mathcal{T}\left(\left(U_{2}^{B^{\prime \prime}}\right)^{T} \cdot \lambda^{B^{\prime \prime}}\right) d U_{2} & =\int \mathcal{T}\left(U_{2}^{B^{\prime \prime}} \cdot \lambda^{B^{\prime \prime}}\right) d U_{2} \\
& =\operatorname{Tr}\left[\lambda^{B^{\prime \prime}}\right] \mathcal{T}\left(\pi^{B^{\prime \prime}}\right)
\end{aligned}
$$

Taking trace on both sides

$$
\operatorname{Tr}\left[\int \mathcal{T}\left(\left(U_{2}^{B^{\prime \prime}}\right)^{T} \cdot \lambda^{B^{\prime \prime}}\right) d U_{2}\right]=2^{-H_{\min }^{\epsilon}\left(A^{\prime \prime} \mid B^{\prime \prime}\right)_{\sigma}}
$$

where the last equality stems from the fact that for $\mathcal{T}$, property 2 holds with $\delta=0$. Next, from the fact that $\tilde{\sigma}-\sigma$ is Hermitian, we can write $\tilde{\sigma}-\sigma=\Delta_{+}-\Delta_{-}$where $\Delta_{ \pm}$are positive semidefinite matrices with disjoint support. This implies that

$$
\begin{aligned}
\|\tilde{\sigma}-\sigma\|_{1} & =\operatorname{Tr}\left[\Delta_{+}\right]+\operatorname{Tr}\left[\Delta_{-}\right] \\
& \leq 2 \epsilon
\end{aligned}
$$

then

$$
\begin{aligned}
\int\left\|\mathcal{T}\left(\left(U_{2}^{B^{\prime \prime}}\right)^{T} \cdot \tilde{\sigma}\right)-\mathcal{T}\left(\left(U_{2}^{B^{\prime \prime}}\right)^{T} \cdot \sigma\right)\right\|_{1} d U_{2} & =\int\left\|\mathcal{T}\left(U_{2}^{T} \cdot\left(\Delta_{+}-\Delta_{-}\right)\right)\right\|_{1} d U_{2} \\
& \leq \int \operatorname{Tr}\left[\mathcal{T}\left(U_{2}^{T} \cdot\left(\Delta_{+}\right)\right] d U_{2}+\int \operatorname{Tr}\left[\mathcal{T}\left(U_{2}^{T} \cdot\left(\Delta_{-}\right)\right)\right] d U_{2}\right. \\
& =\left(\operatorname{Tr}\left[\Delta_{+}\right]+\operatorname{Tr}\left[\Delta_{-}\right]\right) \operatorname{Tr}\left[\mathcal{T}\left(\pi^{B^{\prime \prime}}\right)\right] \\
& \leq 4 \epsilon
\end{aligned}
$$


This directly implies that the average purified distance

$$
\int P\left(\mathcal{T}\left(\left(U_{2}^{B^{\prime \prime}}\right)^{T} \cdot \tilde{\sigma}\right), \mathcal{T}\left(\left(U_{2}^{B^{\prime \prime}}\right)^{T} \cdot \sigma\right)\right) d U_{2} \leq 2 \sqrt{\epsilon}
$$

Define the random variables

$$
\begin{aligned}
& X_{1}:=\operatorname{Tr}\left[\mathcal{T}\left(\left(U_{2}^{B^{\prime \prime}}\right)^{T} \cdot \lambda^{B^{\prime \prime}}\right)\right] \\
& X_{2}:=P\left(\mathcal{T}\left(\left(U_{2}^{B^{\prime \prime}}\right)^{T} \cdot \tilde{\sigma}\right), \mathcal{T}\left(\left(U_{2}^{B^{\prime \prime}}\right)^{T} \cdot \sigma\right)\right)
\end{aligned}
$$

Then, by a union bound and Markov's inequality,

$$
\operatorname{Pr}\left[X_{1} \geq 3 \cdot 2^{-H_{\min }^{\epsilon}\left(A^{\prime \prime} \mid B^{\prime \prime}\right)_{\sigma}} \cup X_{2} \geq 6 \sqrt{\epsilon}\right] \leq \frac{2}{3}
$$

This implies that, there exists a subset of the unitary group over $B^{\prime \prime}$ of probability at least $\frac{1}{3}$ such that, for choices of $U_{2}$ in this set,

$$
H_{\min }^{\epsilon}\left(A^{\prime \prime} \mid R_{2}\right)_{\omega\left(U_{2}\right)} \geq H_{\min }^{\frac{\epsilon^{2}}{36}}\left(A^{\prime \prime} \mid B^{\prime \prime}\right)_{\sigma}-\log 3
$$

As a demonstration of how we will use Theorem 2.16, consider the followin lemma.

Lemma 2.17 Given the intermediary state $\left|\omega\left(U_{2}\right)\right\rangle^{A^{\prime \prime} A^{\prime} B^{\prime} R_{2}}$ and the channel $\mathcal{N}^{A^{\prime} B^{\prime} \rightarrow C}$ with Stinespring dilation $\mathcal{U}^{A^{\prime} B^{\prime} \rightarrow C E}$, and setting the measure $\mu$ to be the Haar measure over the unitary group corresponding to the system $B^{\prime \prime}$, the following holds true with constant probability over the choices of $U_{1}$ and $U_{2}$

$$
\begin{aligned}
& \|\left(\left|A^{\prime \prime}\right| \operatorname{Tr}_{C} \mathcal{U}_{\mathcal{N}} \text { op }^{A^{\prime \prime} \rightarrow A^{\prime} B^{\prime} R_{2}}\left(\omega\left(U_{2}\right)\right) U_{1} \cdot \psi^{A^{\prime \prime} R_{1}}\right)^{R_{1} R_{2} E}-\psi^{R_{1}} \otimes \omega\left(U_{1}\right)^{R_{2} E} \|_{1} \\
& \leq 2^{\frac{1}{2} H_{\min }^{\frac{\epsilon^{2}}{4 k^{2}}}\left(A^{\prime \prime} \mid B^{\prime \prime} E\right)_{\sigma}-\frac{1}{2} H_{\min }^{\epsilon}\left(A^{\prime \prime} \mid R_{1}\right)_{\psi}+\log k}+8 k \epsilon
\end{aligned}
$$

where $k$ is a some positive integer.

Proof:[Proof of Theorem 2.17] We will first apply the smooth single sender decoupling theorem to the quantity on the left in the theorem statement:

$$
\begin{aligned}
& \int \|\left(\left|A^{\prime \prime}\right| \operatorname{Tr}_{C} \mathcal{U}_{\mathcal{N}} \text { op }^{A^{\prime \prime} \rightarrow A^{\prime} B^{\prime} R_{2}}\left(\omega\left(U_{2}\right)\right) U_{1} \cdot \psi^{A^{\prime \prime} R_{1}}\right)^{R_{1} R_{2} E}-\psi^{R_{1}} \otimes \omega\left(U_{2}\right)^{R_{2} E} \|_{1} d U_{1} \\
& \leq 2^{\left.-\frac{1}{2} H_{\min }^{\epsilon}\left(A^{\prime \prime} \mid R_{2} E\right)_{\omega\left(U_{2}\right)}\right)^{-\frac{1}{2}} H_{\min }^{\epsilon}\left(A^{\prime \prime} \mid R_{1}\right)_{\psi}}+8 \epsilon
\end{aligned}
$$

We will now invoke the arguments of Theorem 2.16, with some tweaks. We work with the same almost CPTP map $\mathcal{T}^{B^{\prime \prime} \rightarrow R_{2}}$. Carrying forward the notation of Theorem 2.16 in the natural way, notice that

$$
\begin{aligned}
\int \mathcal{T}\left(\left(U_{2}^{B^{\prime \prime}}\right)^{T} \cdot \lambda^{B^{\prime \prime} E}\right) d U_{2} & =\int \mathcal{T}\left(U_{2}^{B^{\prime \prime}} \cdot \lambda^{B^{\prime \prime} E}\right) d U_{2} \\
& =\mathcal{T}\left(\pi^{B^{\prime \prime}}\right) \otimes \lambda^{E}
\end{aligned}
$$


Taking trace on both sides

$$
\begin{aligned}
\operatorname{Tr}\left[\int \mathcal{T}\left(\left(U_{2}^{B^{\prime \prime}}\right)^{T} \cdot \lambda^{B^{\prime \prime} E}\right) d U_{2}\right] & =\operatorname{Tr}\left[\lambda^{E}\right] \\
& =\operatorname{Tr}\left[\lambda^{B^{\prime \prime} E}\right] \\
& =2^{-H_{\min }^{\epsilon}\left(A^{\prime \prime} \mid B^{\prime \prime} E\right)_{\sigma}}
\end{aligned}
$$

As in Theorem 2.16, define the random variables

1. $X_{1}:=\operatorname{Tr}\left[\mathcal{T}\left(\left(U_{2}^{B^{\prime \prime}}\right)^{T} \cdot \lambda^{B^{\prime \prime} E}\right)\right]$

2. $X_{2}:=P\left(\mathcal{T}\left(\left(U_{2}^{B^{\prime \prime}}\right)^{T} \cdot \tilde{\sigma}\right), \mathcal{T}\left(\left(U_{2}^{B^{\prime \prime}}\right)^{T} \cdot \sigma\right)\right)$

3. $X_{3}:=\|\left(\left|A^{\prime \prime}\right| \operatorname{Tr}_{C} \mathcal{U}_{\mathcal{N}} \text { op }^{A^{\prime \prime} \rightarrow A^{\prime} B^{\prime} R_{2}}\left(\omega\left(U_{2}\right)\right) U_{1} \cdot \psi^{A^{\prime \prime} R_{1}}\right)^{R_{1} R_{2} E}-\psi^{R_{1}} \otimes \omega\left(U_{2}\right)^{R_{2} E} \|_{1}$

Let $k \in \mathbb{N}$ be some positive integer $\geq 4$. Then, repeating the arguments in Theorem 2.16, we conclude that there exists a set of probability at least $1-\frac{3}{k}$ over choices of $U_{1}$ and $U_{2}$ such that the following holds:

$$
\begin{aligned}
& \|\left(\left|A^{\prime \prime}\right| \operatorname{Tr}_{C} \mathcal{U}_{\mathcal{N}} \text { op }^{A^{\prime \prime} \rightarrow A^{\prime} B^{\prime} R_{2}}\left(\omega\left(U_{2}\right)\right) U_{1} \cdot \psi^{A^{\prime \prime} R_{1}}\right)^{R_{1} R_{2} E}-\psi^{R_{1}} \otimes \omega\left(U_{2}\right)^{R_{2} E} \|_{1} \\
& \leq 2^{\frac{1}{2} H_{\text {min }}^{\frac{\epsilon^{2}}{4 k^{2}}}\left(A^{\prime \prime} \mid B^{\prime \prime} E\right)_{\sigma}-\frac{1}{2} H_{\min }^{\epsilon}\left(A^{\prime \prime} \mid R_{1}\right)_{\psi}+\log k}+8 k \epsilon
\end{aligned}
$$

A special case which will be of importance to us is when the measure $\mu$ is a product of 2 Haar

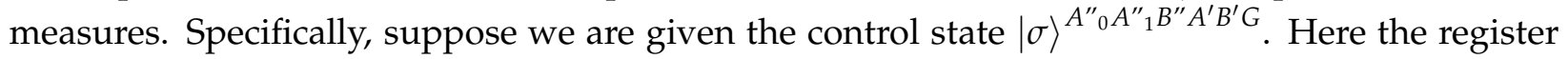
$G$ is present to ensure that $\sigma$ is pure. We will see later that $G$ will not make an appearance in our protocol. Given (Stinespring dilation of) the channel $\mathcal{U}_{\mathcal{N}}^{A^{\prime} B^{\prime} \rightarrow C E}$, let $|\psi\rangle^{R_{1} A^{\prime \prime}}|\eta\rangle^{R_{0} A^{\prime \prime}{ }_{0}}$ and $|\varphi\rangle^{R_{2} B^{\prime \prime}}$ be the transmission states. We define the intermediary state

$$
\left|\omega\left(U_{1}, U_{2}\right)\right\rangle^{A^{\prime \prime}{ }_{0} A^{\prime} B^{\prime} R_{1} R_{2} G}:=\sqrt{A^{\prime \prime}{ }_{1} B^{\prime \prime}}\left(\text { op }^{A^{\prime \prime}{ }_{1} B^{\prime \prime} \rightarrow A^{\prime \prime}{ }_{0} A^{\prime} B^{\prime} G}(\sigma)\left(U_{1}^{A^{\prime \prime}{ }_{1}} \otimes U_{2}^{A^{\prime \prime}{ }_{2}}\right)|\psi\rangle^{A^{\prime \prime}{ }_{1} R_{2}}|\varphi\rangle^{A^{\prime \prime}{ }_{2} R_{2}}\right)
$$

where $U_{1}$ and $U_{2}$ are independent Haar random unitaries.

Lemma 2.18 Given the intermediary state $\left|\omega\left(U_{1}, U_{2}\right)\right\rangle^{A^{\prime \prime}{ }_{0} A^{\prime} B^{\prime} R_{1} R_{2} G}$ and the channel $\mathcal{U}^{A^{\prime} B^{\prime} \rightarrow C E}$, and setting the measure $\mu$ to be the product of 2 Haar measures over the systems $A^{\prime \prime}{ }_{1}$ and $B^{\prime \prime}$, the following holds true with constant probability over the choices of $U_{1}, U_{2}$ and $U_{0}$

$$
\begin{aligned}
& \left\|\left(\left|A^{\prime \prime}{ }_{0}\right| \operatorname{Tr}_{C} \mathcal{U}_{\mathcal{N}} \mathrm{op}^{A^{\prime \prime}{ }_{0} \rightarrow A^{\prime} B^{\prime} R_{1} R_{2} G}\left(\omega\left(U_{1}, U_{2}\right)\right) U_{0} \cdot \eta^{R_{0} A^{\prime \prime}}\right)^{R_{0} R_{1} R_{2} G E}-\eta_{1}^{R_{0}} \otimes \omega\left(U_{1}, U_{2}\right)^{R_{1} R_{2} G E}\right\|_{1} \\
& \leq k \cdot 2^{-\frac{1}{2} H_{\min }^{\epsilon}\left(A^{\prime \prime}{ }_{0} \mid R_{0}\right)_{\eta}-\frac{1}{2} H_{\min }^{\frac{\epsilon}{4 k^{2}}}\left(A^{\prime \prime}{ }_{0} \mid A^{\prime \prime}{ }_{1} B^{\prime \prime} G E\right)_{\sigma}}+8 k \epsilon
\end{aligned}
$$

where $U_{0}$ is a Haar random unitary and $k$ is a some positive integer. 
Proof:[Proof of Theorem 2.18] As before, the single sender decoupling theorem tells us that

$$
\begin{aligned}
& \|\left(\left|A^{\prime \prime}{ }_{0}\right| \operatorname{Tr}_{C} \mathcal{U}_{\mathcal{N}} \text { op }^{A^{\prime \prime}{ }_{0} \rightarrow A^{\prime} B^{\prime} R_{1} R_{2} G}\left(\omega\left(U_{1}, U_{2}\right)\right) U_{0} \cdot \eta^{R_{0} A^{\prime \prime}{ }_{0}}\right)^{R_{0} R_{1} R_{2} G E}-\eta_{1}^{R_{0}} \otimes \omega\left(U_{1}, U_{2}\right)^{R_{1} R_{2} G E} \|_{1} \\
& \leq 2^{-\frac{1}{2} H_{\min }^{\epsilon}\left(A^{\prime \prime}{ }_{0} \mid R_{0}\right)_{\eta}-\frac{1}{2} H_{\min }^{\epsilon}\left(A^{\prime \prime}{ }_{0} \mid R_{1} R_{2} G E\right)_{\omega\left(U_{1}, U_{2}\right)}+8 \epsilon}
\end{aligned}
$$

Define the map

$$
\mathcal{T}^{A^{\prime \prime}{ }_{1} B^{\prime \prime} \rightarrow R_{1} R_{2}}(\xi):=\left|A^{\prime \prime}{ }_{1} B^{\prime \prime}\right|\left(\operatorname{op}^{A^{\prime \prime}{ }_{1} B^{\prime \prime} \rightarrow R_{1} R_{2}}(|\psi\rangle|\varphi\rangle) \cdot \xi\right)
$$

First, recall the following properties of Haar integration

1. $\int U_{1}^{A} \otimes U_{2}^{B} \cdot \rho^{A B} d U_{1} d U_{2}=\operatorname{Tr}\left[\rho^{A B}\right] \pi^{A B}$

2. $\int U_{1}^{A} \otimes U_{2}^{B} \otimes I^{C} \cdot \rho^{A B C} d U_{1} d U_{2}=\pi^{A B} \otimes \rho^{C}$

It is now easy to verify that $\mathcal{T}$ is indeed an almost CPTP. The first two properties can be shown to be true using reasoning similar to that used in Theorem 2.16. Finally, using property 1 of double Haar integration above, one can immediately see that

$$
\int \mathcal{T}\left(U_{1} \otimes U_{2} \cdot \xi^{A^{\prime \prime}{ }_{1} B^{\prime \prime}}\right) d U_{1} d U_{2}=\operatorname{Tr}[\xi] \mathcal{T}\left(\pi^{A^{\prime \prime} B^{\prime \prime}}\right)
$$

Next suppose $\tilde{\sigma}^{A{ }^{\prime \prime}{ }_{1}{ }^{\prime \prime}{ }_{0} B^{\prime \prime} C E G}$ be a state such that $H_{\min }^{\epsilon}\left(A^{\prime \prime}{ }_{0} \mid A^{\prime \prime}{ }_{1} B^{\prime \prime} G E\right)_{\sigma}=H_{\min }\left(A^{\prime \prime}{ }_{0} \mid A^{\prime \prime}{ }_{1} B^{\prime \prime} G E\right)_{\tilde{\sigma}}$ where $\left\|\tilde{\sigma}-\mathcal{U}_{\mathcal{N}} \cdot \sigma\right\|_{1} \leq 2 \epsilon$. Let $\lambda^{A^{\prime \prime}{ }_{1} B^{\prime \prime} G E}$ be a positive semidefinite matrix such that

$$
\operatorname{Tr}[\lambda]=2^{-H_{\min }^{\epsilon}\left(A_{0}{ }_{0} \mid A^{\prime \prime}{ }_{1} B^{\prime \prime} G E\right)_{\sigma}}
$$

and

$$
\tilde{\sigma}^{A{ }^{\prime \prime} A^{\prime \prime}{ }_{1} B^{\prime \prime} G E} \leq \mathbb{I}^{A^{\prime \prime} 0} \otimes \lambda^{A^{\prime \prime} B^{\prime \prime} G E}
$$

Then, it holds that

$$
\begin{aligned}
\operatorname{Tr}\left[\int \mathcal{T}\left(\left(U_{1}^{A^{\prime \prime}{ }_{1}} \otimes U_{2}^{B^{\prime \prime}}\right)^{T} \cdot \lambda^{A^{\prime \prime}{ }_{1} B^{\prime \prime} G E} d U_{1} d U_{2}\right]\right. & =\operatorname{Tr}\left[\mathcal{T}\left(\pi^{A^{\prime \prime}{ }_{1} B^{\prime \prime}}\right) \otimes \lambda^{G E}\right] \\
& =2^{-H_{\min }^{\epsilon}\left(A^{\prime \prime}{ }_{0} \mid A^{\prime \prime}{ }_{1} B^{\prime \prime} G E\right)_{\sigma}}
\end{aligned}
$$

Similarly, one can show that

$$
\int P\left(\mathcal{T}\left(\left(U_{1}^{A^{\prime \prime}}{ }_{1} \otimes U_{2}^{B^{\prime \prime}}\right)^{T} \cdot \tilde{\sigma}\right), \mathcal{T}\left(\left(\left(U_{1}^{A^{\prime \prime}}{ }_{1} \otimes U_{2}^{B^{\prime \prime}}\right)^{T} \mathcal{U}_{\mathcal{N}} \cdot \sigma\right)\right) d U_{1} d U_{2} \leq 2 \sqrt{\epsilon}\right.
$$

Finally, noting that $\mathcal{T}\left(\left(U_{1}^{A^{\prime \prime}}{ }_{1} \otimes U_{2}^{B^{\prime \prime}}\right)^{T} \cdot \sigma\right)=\omega\left(U_{1}, U_{2}\right)$ and using the arguments in Theorem 2.17 we conclude the proof. 


\section{Rate Splitting for Point to Point Channels}

\subsection{Rate Splitting in the Classical Regime}

In this section we briefly review the idea of rate splitting, as detailed in [GRUW01]. Consider the classical point to point channel $\left(\mathcal{A}, P_{B \mid A}, \mathcal{B}\right)$ between Alice and Bob and let $P_{A}$ be the input distribution that maximises $I(A: B)$. The idea is to split Alice into two independent senders, Alice $_{0}$ and Alice ${ }_{1}$ and then have Bob decode their messages via a successive cancellation strategy. To do this, one shows the existence of a family of triples $\left(P_{U}^{\theta}, P_{V}^{\theta}, f\right)$ where $\theta$ is a parameter in $[0,1], P_{U}^{\theta}$ and $P_{V}^{\theta}$ are independent distributions on $\mathcal{A}$ and $f$ is a deterministic function, such that $f\left(U^{\theta}, V^{\theta}\right) \sim P_{A}$. Furthermore, appealing to the properties of the mutual information one can show that

$$
I(A: B)=I\left(U^{\theta} V^{\theta}: B\right)=I\left(U^{\theta}: B\right)+I\left(V^{\theta}: B U^{\theta}\right)
$$

From the above discussion it is clear that a simple encoding-decoding strategy is as follows : Alice $_{0}$ uses a code of rate $I\left(U^{\theta}: B\right)$ regarding Alice ${ }_{1}$ as noise and Alice ${ }_{1}$ uses a code of rate $I\left(V^{\theta}: B U^{\theta}\right)$ regarding Alice ${ }_{0}$ as side information at the receiver. Bob decodes via successive cancellation. Finally, one can show that $\left(I\left(U^{\theta}: B\right), I\left(V^{\theta}: B U^{\theta}\right)\right)$ is a continuous function in $\theta \in[0,1]$ and traces out the straight line joining the points $(0, I(A: B))$ and $(I(A: B), 0)$ due to the chain rule of mutual information with equality. With this construction in hand, one can design an encoding and decoding scheme for the classical MAC without appealing to time sharing or jointly typical simultaneous decoding. Firstly, split Alice into the two users Alice $_{0}$ and Alice 1 by the construction above. Then Charlie does a successive cancellation decoding for this 3 sender MAC: first decode Alice ${ }_{0}$ 's message treating the other senders as noise, then decode Bob's message regarding Alice ${ }_{0}$ 's message as side information and Alice ${ }_{1}$ as noise, and finally decode Alice ${ }_{1}$ 's message regarding Bob's and Alice ${ }_{0}$ 's message as side information. Thus three point to point channel decodings are done by Charlie in order to decode the sent messages at the rate triple $\left(I\left(U^{\theta}: C\right), I\left(C: B U^{\theta}\right), I\left(V^{\theta}: C B U^{\theta}\right)\right)$. Notice that, all points in the dominant face of the achievable region in Figure 1 can be achieved in this way due to continuity as $\theta$ varies from 0 to 1 . Also observe that the split of Alice depends on $\theta$.

The triple $\left(f, P_{U}^{\theta}, P_{V}^{\theta}\right)$ with respect to the distribution $P_{A}$ is called a split of $P_{A}$. That such a triple exists is given by the following fact:

Fact 3.1 Given a distribution $P_{A}$ on the set $\mathcal{A}$, there exist two distributions $P_{U}^{\theta}$ and $P_{V}^{\theta}$ (both defined on $\mathcal{A})$, parameter $\theta \in[0,1]$ and a function $f: \mathcal{A} \times \mathcal{A} \rightarrow \mathcal{A}$ such that the following hold true :

1. $f(U, V) \sim P_{A}$

2. For fixed values of $x$ and $u, P_{f(U, V) \mid U}^{\theta}(a \mid u)$ is a continuous function of $\theta$.

3. For $\theta=0, P_{f(U, V) \mid U}^{\theta}(a \mid u)=P_{A}(a)$.

4. For $\theta=1$, and all $u \in \mathcal{A}, P_{f(U, V) \mid U}^{\theta}(a \mid u)$ puts all its mass on one element.

Proof: We demonstrate an explicit construction, as shown in [GRUW01]. Assume that $\mathcal{A}$ is an ordered set. We describe the distribution in terms of distribution functions, for which we use the 
letter $F$ along with the appropriate subscript. Then, define, for all $i \in \mathcal{A}$ :

$$
\begin{aligned}
& F_{U}^{\theta}(i):=\theta F_{A}+1-\theta \\
& F_{V}^{\theta}(i):=\frac{F_{A}(i)}{F_{U}^{\theta}(i)} \\
& f(u, v):=\max \{u, v\} \quad \forall u, v \in \mathcal{A}
\end{aligned}
$$

It is easy to check the triple defined above satisfies all the properties in Theorem 3.1. The interested reader may look at [GRUW01] for details.

\subsection{Entanglement Transmission}

We first describe the task of entanglement transmission over a quantum channel. Unlike its classical counterpart, there are multiple seemingly disparate information transmission tasks that one can consider to define the quantum capacity of a quantum channel. However, it was shown in [KW03], that these definitions are equivalent. In this paper, we will consider the task of entanglement transmission.

Alice has access to the $A$ system of the EPR state $|\Phi\rangle^{R A}$, where $|R|=|A|$. Alice possess a decoding CРTP map $\mathcal{E}^{A \rightarrow A^{\prime}}$ such that, after acting this map on the system $A$, Alice sends the $A^{\prime}$ part of the pure state $(\mathcal{E} \cdot \Phi)^{R A^{\prime}}$ through the channel $\mathcal{N}^{A^{\prime} \rightarrow B}$. Finally, Bob applies a decoding map $\mathcal{D}^{B \rightarrow \hat{A}}$ on his system $B$, such that the final state he shares with $R$ is close to the EPR state $|\Phi\rangle^{R A}$.

Formally, a $(Q, \epsilon)$ entanglement generation code consists of an encoder $\mathcal{E}^{A \rightarrow A^{\prime}}$ and a decoding CPTP $\mathcal{D}^{B \rightarrow \hat{A}}$ such that

$$
\begin{array}{r}
|\Phi\rangle^{R A}=\frac{1}{\sqrt{2^{Q}}} \sum_{i=1}^{2^{Q}}|i\rangle^{R}|i\rangle^{A} \\
F\left(|\Phi\rangle^{R A}, \mathbb{I}^{R} \otimes(\mathcal{D} \circ \mathcal{N} \circ \mathcal{E})\left(\Phi^{R A}\right)\right) \geq 1-\epsilon
\end{array}
$$

A rate $Q$ is achievable for the channel $\mathcal{N}$ is there exists a $(Q, \epsilon)$ code for $\mathcal{N}$.

In fact we consider the following more general situation: Alice and Bob share the pure state $\psi^{A B R}$ where Alice holds $A$, Bob holds $B$ and $R$ is the reference. Alice wants to send her share to Bob through a single use of the channel $\mathcal{N}^{A^{\prime} \rightarrow C}$. To do this, one needs to show the existence of an encoder decoder pair $\mathcal{E}^{A \rightarrow A^{\prime}}$ and $\mathcal{D}^{C B \rightarrow \hat{A} B}$ such that

$$
\|\mathcal{D} \circ \mathcal{N} \circ \mathcal{E}(\psi)-\psi\|_{1} \leq \epsilon
$$

Note that in this situation Bob can potentially utilise the correlations present in the system $B$ for decoding, to boost the rate of transmission.

To derive an entanglement transmission protocol out of this, we set $\psi^{A B R}$ to be the state $\Phi^{R M} \otimes$ $\Phi^{\tilde{A} B}$, where the registers $M \tilde{A}$ play the role of $A$ in Theorem 2.11. Notice that $\Phi^{\tilde{A} B}$ is essentially shared entanglement between Alice and Bob, and changing the rank of $\tilde{A}$ essentially allows us to control the amount of shared entanglement available for use in the protocol. Thus, we call this situation entanglement transmission with rate limited entanglement assistance or partial entanglement assisted transmission protocol. [Dup10]. 
We say that a rate pair $(Q, E)$ ( where $Q$ denotes the rank of the EPR state to be transmitted, in this case $|R|=|M|$ and $E$ denotes the amount of pre-shared entanglement available for use during the protocol) is $\epsilon$-achievable if there exists an encoder decoder pair such that

$$
\left\|\mathcal{D} \circ \mathcal{N} \circ \mathcal{E}\left(\Phi^{R M} \otimes \Phi^{\tilde{A} B}\right)-\Phi^{R M}\right\|_{1} \leq \epsilon
$$

The rate $Q$ is achievable for transmission with entanglement assistance if there exists $E \geq 0$ such that $(Q, E)$ is $\epsilon$ achievable, and it is achievable for unassisted transmission if $(Q, 0)$ is $\epsilon$ achievable.

The idea behind proving that such an encoder decoder pair exist is to consider the complementary channel $\mathcal{N}^{A^{\prime} \rightarrow E}$. Suppose one can show that

$$
\overline{\mathcal{N}}^{A^{\prime} \rightarrow E} \circ \mathcal{E}^{A \rightarrow A^{\prime}}\left(\psi^{A R}\right) \approx \overline{\mathcal{N}} \circ \mathcal{E}\left(\psi^{A}\right) \otimes \psi^{R}
$$

Intuitively this would imply that the original channel preserves correlations perfectly, and then the existence of a decoder can be inferred by appealing to Uhlmann's theorem. The main work then goes towards proving the existence of an encoder which has this property.

To do this, consider the state $\left|\omega^{A^{\prime \prime} C E}\right\rangle:=\mathcal{U}_{\mathcal{N}}^{A^{\prime}}|\sigma\rangle^{A^{\prime \prime} A^{\prime}}$. The state $\omega$ will act as our control state. We will consider a randomized encoder, augmented by a Haar random unitary. This encoder takes the follwoing form :

$$
\mathcal{E}_{\mathrm{RAND} \_ \text {ENC }}^{A \rightarrow A^{\prime}}\left(\psi^{A R}\right):=\mathrm{op}^{A^{\prime \prime} \rightarrow A^{\prime}}\left(|\sigma\rangle^{A^{\prime \prime} A^{\prime}}\right) U_{\mathrm{RAND}}^{A^{\prime \prime}} W^{A \rightarrow A^{\prime \prime}} \cdot \psi^{A R}
$$

where $W$ is some isometric embedding from the space $A$ to $A^{\prime \prime}$ and $U_{\mathrm{RAND}}$ is a Haar random unitary.

It can be shown that averaging over all unitaries, this randomized encoder satisfies the following properties :

1. There exists a fixed isometric encoder which does almost as well as the randomized encoder. The error in the performance of the fixed isometric encoding and the randomized encoder is measure by the quantity $H_{\max }^{\epsilon}(A)_{\psi}-H_{2}^{\epsilon}\left(A^{\prime \prime}\right)_{\Omega}$ i.e. how close the input distribution $\psi^{A}$ is to the target distribution $\omega^{A^{\prime \prime}}$.

2. The action of the complementary channel and the randomized encoder nearly decouples the systems $E$ and $R$. The accuracy of the decoupling is measured by the quantity $-H_{2}^{\epsilon}\left(A^{\prime \prime} \mid E\right)_{\Omega}-$ $H_{2}^{\epsilon}(A \mid R)_{\psi}$.

Collating the two properties together one can infer the existence of an isometric encoder, and by a subsequent application of Uhlmann's theorem, a decoder such that Alice can send the register $A$ through the channel with high fidelity, given that the entropic constraints are satisfied. One should note that both desired properties above, along with the entropic conditions are given by direct applications of the single user decoupling theorem. The above discussion can be encapsulated by the following fact:

Fact 3.2 For any quantum channel $\mathcal{N}^{A^{\prime} \rightarrow C}$ and pure state $\sigma^{A^{\prime \prime}} A^{\prime}$ where $A^{\prime \prime}$ and $A^{\prime}$ are isomorphic, the rate pair $(Q, E)$ is $\epsilon$ achievable for quantum transmission with rate limited assistance through $\mathcal{N}$ if

$$
\begin{aligned}
& Q+E<H_{2}^{\epsilon}\left(A^{\prime \prime}\right)_{\Omega} \\
& Q-E<H_{2}^{\epsilon}\left(A^{\prime \prime} \mid E\right)_{\Omega}
\end{aligned}
$$


where we have suppressed some additive error terms in terms of $\log \epsilon$ in the bounds above. A more precise version of this theorem is stated as Theorem 2.11. Notice that, to get the unassisted entanglement transmission rate, one need only set $E$ to 0 . This implies that the unassisted rate is $\approx H_{2}^{\epsilon}\left(A^{\prime \prime} \mid E\right)_{\mathcal{U}_{\mathcal{N}} \Omega}$ after suppressing the additive log terms.

\subsection{Rate Splitting in the Quantum Case}

To describe rate splitting in the entanglement transmission scenario, we will define an abstract splitting scheme with some properties of interest:

Definition 3.3 Splitting Scheme Given a control state $|\sigma\rangle^{A^{\prime \prime} A^{\prime} B^{\prime \prime} B^{\prime}}=|\Omega\rangle^{A^{\prime \prime} A^{\prime}}|\Delta\rangle^{B^{\prime \prime} B^{\prime}}$ and systems $A^{\prime \prime}{ }_{0}$ and $A^{\prime \prime}{ }_{1}$ such that $A^{\prime \prime} \cong A^{\prime \prime}{ }_{1} \cong A^{\prime \prime}{ }_{0}$, we define a splitting scheme to be a family of isometric embeddings $U_{\theta^{A \prime} \rightarrow A^{\prime \prime}{ }_{0} A^{\prime \prime}}$ parametrized by a variable $\theta \in[0,1]$, such that:

1. For all $\theta, \theta^{\prime} \in[0,1]$ and $\epsilon>0$ there exists $\delta>0$ such that whenever $\left|\theta-\theta^{\prime}\right| \leq \delta,\left\|U_{\theta} \cdot \sigma-U_{\theta^{\prime}} \cdot \sigma\right\|_{1} \leq$ $\epsilon$

2. Given any channel $\mathcal{N}^{A^{\prime} B^{\prime} \rightarrow C}$ and its Stinespring dilation $\mathcal{U}_{\mathcal{N}}^{A^{\prime} B^{\prime} \rightarrow C E}$,

$$
I\left(A^{\prime \prime}{ }_{0} B^{\prime \prime}>C\right)_{\sigma_{0}}=I\left(A^{\prime \prime}{ }_{1} B^{\prime \prime}>C\right)_{\sigma_{1}}=I\left(A^{\prime \prime} B^{\prime \prime}>C\right)_{\sigma}
$$

where $\sigma_{0}:=U_{0} \cdot \sigma$ and $\sigma_{1}:=U_{1} \cdot \sigma$.

Notice that we have define the splitting scheme with respect to the more general control state $|\sigma\rangle^{A^{\prime \prime} A^{\prime} B^{\prime \prime} B^{\prime}}=|\Omega\rangle^{A^{\prime \prime} A^{\prime}}|\Delta\rangle^{B^{\prime \prime} B^{\prime}}$. This will be useful when we describe the splitting protocol for more general multiterminal channels. For the purposed of this section, where we only demonstrate splitting for the point to point channel, one may simply ignore the state $|\Delta\rangle$. Also note that the invariants in the splitting scheme are specified in terms of the coherent information. A more general definition would be to specify the invariants in terms of the smooth min entropy. We work with this more general definition.

We will first give an overview of the strategy for the unassisted case. We will then state and prove the main technical lemma of this section, Theorem 3.4. The ideas in used in proving this lemma will generalise easily to the setting of the multiterminal channels such as the QMAC and the QIC.

We will emulate the strategy outlined in Section 3.1 for a bipartite pure quantum state $|\Omega\rangle^{A^{\prime \prime} A^{\prime}}:=$ $\sum_{a^{\prime \prime} \in \mathcal{A}^{\prime \prime}} \sqrt{P_{A^{\prime \prime}}(a)}|a\rangle^{A^{\prime \prime}}\left|\zeta_{a}\right\rangle^{A^{\prime}}$, where $\left|a^{\prime \prime}\right\rangle$ runs over the computational basis of $A^{\prime \prime}$ and $P_{A^{\prime \prime}}$ is a probability distribution on the basis set $\mathcal{A}^{\prime \prime}$. We split the system $A^{\prime \prime}$ into two registers $A_{0}^{\prime \prime}$ and $A_{1}^{\prime \prime}$

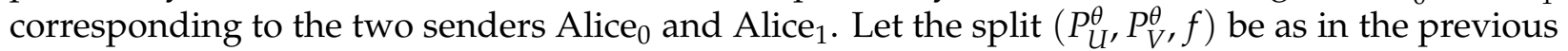
subsection. Define the isometric embedding $U_{\mathrm{SPLIT}}(\theta)^{A^{\prime \prime} \rightarrow A_{0}^{\prime \prime} A_{1}^{\prime \prime}}$ as follows:

$$
\sqrt{P_{A}(a)}|a\rangle \stackrel{A^{\prime \prime}}{\stackrel{U_{\text {SPLIT }}(\theta)}{\longmapsto}} \sum_{(u, v) \in f^{-1}\left(a^{\prime \prime}\right)} \sqrt{P_{U}^{\theta}(u) P_{V}^{\theta}(v)}|u\rangle^{A_{0}^{\prime \prime}}|v\rangle^{A_{1}^{\prime \prime}}
$$

and $|\Omega(\theta)\rangle^{A_{0}^{\prime \prime} A_{1}^{\prime \prime} A^{\prime}}:=U_{\text {SPLit }}(\theta)|\Omega\rangle^{A^{\prime \prime} A^{\prime}}$.

We now pass the system $A^{\prime}$ through a point to point channel $\mathcal{N}^{A^{\prime} \rightarrow B}$ and obtain the quantum state $|\Omega(\theta)\rangle_{0}^{A_{1}^{\prime \prime} A_{1}^{\prime \prime} B}$. By unitary invariance, $I_{2}^{\epsilon}\left(A^{\prime \prime}>B\right)_{\Omega}=I_{2}^{\epsilon}\left(A_{0}^{\prime \prime} A_{1}^{\prime \prime}>B\right)_{\Omega(\theta)}$. From the works of [Dup10, SDTR13] applied to transmission of quantum information over one-shot unassisted 
point to point quantum channels, we first realise that Bob can decode Alice ${ }_{0}$ 's quantum message at the rate of $I_{2}^{\epsilon^{2} / 800}\left(A_{0}^{\prime \prime}>B\right)_{\Omega(\theta)}-O\left(\log \epsilon^{-1}\right)$ with error at most $O(\sqrt{\epsilon})$. Then, employing the successive cancellation methods of Yard et al. [YDH05] Bob can decode Alice ${ }_{2}$ 's quantum message at the rate of $I_{2}^{\epsilon^{2} / 800}\left(A_{1}^{\prime \prime}>B A_{0}^{\prime \prime}\right)_{\Omega(\theta)}-O\left(\log \epsilon^{-1}\right)$ with error at most $O(\sqrt{\epsilon})$.

Doing both the steps above requires us to overcome a few technical challenges which we do by defining a notion of almost CPTP maps (see Section 2.6) that should be useful in other situations too, and combining it with another proof technique by Dupuis for the unassisted quantum broadcast channel [Dup10].

We have thus operationally shown the chain rule inequality $I_{\min }^{\epsilon^{2} / 800}\left(A_{1}^{\prime \prime}>B A_{0}^{\prime \prime}\right)_{\Omega(\theta)}+I_{\min }^{\epsilon^{2} / 800}\left(A_{0}^{\prime \prime}>\right.$ $B)_{\Omega(\theta)} \leq I_{\min }^{\epsilon}\left(A_{0}^{\prime \prime} A_{1}^{\prime \prime}>B\right)_{\Omega(\theta)}$. (suppressing the log factors). One can prove this fact independently using the chain rule for smooth min entropies Theorem 2.1. We now see that as $\theta$ varies from 0 to 1 , the point $\left(R_{0}(\theta), R_{1}(\theta)\right)=\left(I_{\min }^{\epsilon^{2} / 800}\left(A_{0}^{\prime \prime}>B\right)_{\Omega(\theta)}, I_{\min }^{\epsilon / 800}\left(A_{1}^{\prime \prime}>B A_{0}^{\prime \prime}\right)_{\Omega(\theta)}\right)$ traces out a continuous curve that lies on or below the line segment joining the point $\left(I_{\min }^{\epsilon}(A>B), 0\right)$ to the point $\left(0, I_{\min }^{\epsilon}(A>B)\right)$ and meets it at its endpoints. The continuity of the curve follows from the continuity of the states and the functionals involved. Continuity of the functionals is implied by Theorem 2.3 whereas continuity of the states is implied by Theorem A.1. These arguments also imply that $U_{\text {SPLIT }}(\theta)$ is indeed a valid splitting scheme.

This rate splitting and successive cancellation idea can now be easily generalised to the unassisted QMAC.

We will now consider the general case, when Bob has side information available at the decoder. Suppose Alice ${ }_{0}$ and Alice ${ }_{1}$ wish to transmit the systems $A_{0}$ and $A_{1}$ of the states $|\eta\rangle^{A_{0} B_{0} R_{0}}$ and $|\psi\rangle^{A_{1} B_{1} R_{1}}$ to Bob. We wish to prove there exists an encoder $\mathcal{E}^{A_{0} A_{1} \rightarrow A^{\prime}}$ and a decoder $\mathcal{C}^{B C_{0} C_{1} \rightarrow A_{0} A_{1}}$ such that

$$
F(\mathcal{C} \circ \mathcal{N} \circ \mathcal{E}(\eta \otimes \psi), \eta \otimes \psi) \geq 1-\epsilon
$$

Given that such an encoder decoder pair exist, set $|\eta\rangle^{A_{0} B_{0} R_{0}} \leftarrow|\Phi\rangle^{M_{0} R_{0}}|\Phi\rangle^{\tilde{A}_{0} B_{0}}$ and $|\psi\rangle^{A_{1} B_{1} R_{1}} \leftarrow$ $|\Phi\rangle^{A_{1} M_{1}}|\Phi\rangle^{\tilde{A}_{1} B_{1}}$. Let $Q_{A_{0}}=\log \left|M_{0}\right|, Q_{A_{1}}=\log \left|M_{2}\right|$. and $E_{A_{0}}=\log \left|B_{0}\right|, E_{A_{1}}=\log \left|B_{1}\right|$. The rates $Q_{A_{0}}, Q_{A_{1}}$ are the entanglement transmission rates of Alice 0 and Alice ${ }_{1}$ and $E_{A_{0}}$ and $E_{A_{1}}$ quantify the amount of pre-shared entanglement available to them before the protocol begins.

We will consider the simpler case, when Alice $_{0}$ does not share any entanglement with Bob, but Alice $_{1}$ does, i.e. the register $C_{0}$ is trivial. We quantify the rates in the following lemmas:

Proposition 3.4 Given the control state $|\Omega\rangle^{A^{\prime \prime} A^{\prime}}$, the point to point quantum channel $\mathcal{N}^{A^{\prime} \rightarrow B}$ and the splitting scheme $U_{\theta}^{A^{\prime \prime}}$, suppose Alice has to send states $|\eta\rangle^{A_{0} R_{0}} \otimes|\psi\rangle^{A_{1} B_{1} R_{1}}$ to Bob, where $A_{0}$ and $A_{1}$ are the message registers and $B_{1}$ models the side information $B o b$ has about the $A_{1} . R_{0}$ and $R_{1}$ are reference systems. We define $\left|\Omega^{\prime}(\theta)\right\rangle^{A^{\prime \prime}{ }_{0} A^{\prime \prime}{ }_{1} A^{\prime}}:=U_{\theta}^{A^{\prime \prime}}|\Omega\rangle^{A^{\prime \prime} A^{\prime}}$ and

$$
\left|\Omega^{\prime}(\theta)\right\rangle^{A^{\prime \prime}{ }_{0}{ }^{\prime \prime}{ }_{1} B E}:=\mathcal{U}_{\mathcal{N}}^{A^{\prime} \rightarrow B E}\left|\Omega^{\prime}(\theta)\right\rangle^{A^{\prime \prime}{ }_{0} A{ }_{1} A^{\prime}}
$$

Then there exist an encoder $\mathcal{E}^{A_{0} A_{1} \rightarrow A^{\prime}}$ and a decoder $\mathcal{C}^{B B_{1} \rightarrow A_{0} A_{1}}$ such that

$$
\left\|\mathcal{C} \circ \mathcal{N} \circ \mathcal{E}\left(\eta^{A_{0} R_{0}} \otimes \psi^{A_{1} B_{1} R_{1}}\right)-\eta^{A_{0} R_{0}} \otimes \psi^{A_{1} B_{1} R_{1}}\right\|_{1} \leq \delta
$$


where $\delta=4 \sqrt{2 \delta_{\mathrm{dec}}(0)}+2 \sqrt{2 \delta_{\mathrm{dec}}(1)}+2 \sqrt{2 \delta_{\mathrm{enc}(0)}+2 \delta_{\mathrm{enc}}(1)}$

and

$$
\begin{aligned}
& \delta_{\mathrm{dec}}(0)=20 \cdot 2^{-\frac{1}{2} H_{2}^{\epsilon}\left(A_{0} \mid R_{0}\right)_{\eta}-\frac{1}{2} H_{\min }^{\epsilon_{0}}\left(A^{\prime \prime}{ }_{0} \mid A^{\prime \prime}{ }_{1} E\right)_{\Omega^{\prime}(\theta)}}+160 \epsilon \\
& \delta_{\mathrm{dec}}(1)=20 \cdot 2^{-\frac{1}{2} H_{2}^{\epsilon}\left(A_{1} \mid R_{1}\right)_{\psi}-\frac{1}{2} H_{\min }^{\epsilon_{0}}\left(A^{\prime \prime}{ }_{1} \mid E\right)_{\Omega^{\prime}(\theta)}+160 \epsilon} \\
& \delta_{\mathrm{enc}}(0)=20 \cdot 2^{\frac{1}{2} H_{\max }^{\epsilon}\left(A_{0}\right)_{\eta}-\frac{1}{2} H_{\min }^{\epsilon_{0}}\left(A^{\prime \prime}{ }_{0} \mid A^{\prime \prime}{ }_{1}\right)_{\Omega^{\prime}(\theta)}+160 \epsilon} \\
& \delta_{\mathrm{enc}}(1)=2^{\frac{1}{2} H_{\max }^{\epsilon}\left(A_{1}\right)_{\psi}-\frac{1}{2} H_{\min }^{\epsilon}\left(A^{\prime \prime}{ }_{1}\right)_{\Omega^{\prime}(\theta)}+8 \epsilon}
\end{aligned}
$$

where $\epsilon_{0}=\frac{\epsilon^{2}}{800}$ for some positive $\epsilon$.

An easy corollary of Theorem 3.4 is the following:

Corollary 3.5 Given the control state $|\Omega\rangle^{A^{\prime \prime} A^{\prime}}$ and the point to point channel $\mathcal{N}^{A^{\prime} \rightarrow B}$, and the splitting scheme $U_{\theta}^{A^{\prime \prime} \rightarrow A^{\prime \prime}{ }_{0}{ }^{\prime \prime}{ }_{1}}$, Alice can transmit EPR states to Bob at the rate $Q_{A_{0}}+Q_{A_{1}}$ given $E_{A_{1}}$ bits of preshared entanglement, with error at most $240 \sqrt{\epsilon}$ whenever

$$
\begin{aligned}
& Q_{A_{0}}<H_{\max }^{\epsilon_{0}}\left(A^{\prime \prime}{ }_{0} \mid A^{\prime \prime}{ }_{1}\right)_{\Omega^{\prime}(\theta)}+\log 4 \epsilon^{2} \\
& Q_{A_{0}}<I_{\min }^{\epsilon_{0}}\left(A^{\prime \prime}{ }_{0}>B\right)_{\mathcal{U}_{\mathcal{N}} \cdot \Omega^{\prime}(\theta)}+\log 4 \epsilon^{2} \\
& Q_{A_{1}}+E_{A_{1}}<H_{\max }^{\epsilon}\left(A^{\prime \prime}{ }_{1}\right)_{\Omega^{\prime}(\theta)}+\log 4 \epsilon^{2} \\
& Q_{A_{1}}-E_{A_{1}}<I_{\min }^{\epsilon_{0}}\left(A^{\prime \prime}{ }_{1}>A^{\prime \prime}{ }_{0} B\right)_{\mathcal{U}_{\mathcal{N}} \cdot \Omega^{\prime}(\theta)}+\log 4 \epsilon^{2}
\end{aligned}
$$

where $\epsilon_{0}=\frac{\epsilon^{2}}{800}$ and $\left|\Omega^{\prime}\right\rangle^{A^{\prime \prime}{ }_{0} A^{\prime \prime}{ }_{1} A^{\prime}}=U_{\theta}^{A^{\prime \prime}}|\Omega\rangle^{A^{\prime \prime} A^{\prime}}$.

Proof:[Proof of Theorem 3.5] We initialise the states $|\eta\rangle^{A_{0} R_{0}}$ and $|\psi\rangle^{A_{1} R_{1} B_{1}}$ as follows

$$
\begin{aligned}
& |\eta\rangle^{A_{0} R_{0}} \leftarrow|\Phi\rangle^{A_{0} R_{0}} \\
& |\psi\rangle^{A_{1} R_{1} B_{1}} \leftarrow|\Phi\rangle^{R_{1} M_{1}}|\Phi\rangle^{\tilde{A}_{1} B_{1}}
\end{aligned}
$$

Here, the registers $M_{1} \tilde{A}_{1}$ play the roles of $A_{1}$, and the notation $\Phi$ is used generically to mean an EPR state. Let

$$
\begin{aligned}
& \left|R_{0}\right|=2^{Q_{A_{0}}} \\
& \left|R_{1}\right|=2^{Q_{A_{1}}} \text { and }\left|B_{1}\right|=2^{E_{A_{1}}}
\end{aligned}
$$

Note that Alice's actual rate $Q_{A}$ is $Q_{A_{0}}+Q_{A_{1}}$. The following relatons are easy to check:

$$
\begin{aligned}
& H_{\max }\left(A_{0}\right)_{\eta}=Q_{A_{0}} \Longrightarrow H_{\max }^{\epsilon}\left(A_{0}\right)_{\eta} \leq Q_{A_{0}} \\
& H_{\max }\left(M_{1} \tilde{A}_{1}\right)_{\psi}=Q_{A_{1}}+E_{A_{1}} \Longrightarrow H_{\max }^{\epsilon}\left(M_{1} \tilde{A}_{1}\right)_{\psi} \leq Q_{A_{1}}+E_{A_{1}} \\
& H_{\min }\left(A_{0} \mid R_{0}\right)_{\eta}=Q_{A_{0}} \Longrightarrow H_{\min }^{\epsilon}\left(A_{0}\right)_{\eta} \geq Q_{A_{0}} \\
& H_{\min }\left(M_{1} \tilde{A}_{1} \mid R_{1}\right)_{\psi}=E_{A_{1}}-Q_{A_{1}} \Longrightarrow H_{\min }^{\epsilon}\left(M_{1} \tilde{A}_{1} \mid R_{1}\right)_{\psi} \geq E_{A_{1}}-Q_{A_{1}}
\end{aligned}
$$


Then, from Theorem 3.4, we set

$$
\begin{aligned}
& \delta_{\text {dec }}(0)<200 \epsilon \\
& \delta_{\text {dec }}(1)<200 \epsilon \\
& \delta_{\text {enc }}(0)<200 \epsilon \\
& \delta_{\text {enc }}(1)<16 \epsilon
\end{aligned}
$$

Pluggin in these numbers in the bounds shown in Theorem 3.4 completes the proof.

Proof:[Proof of Theorem 3.4]

Consider the randomised encoder

$$
\mathcal{E}_{\mathrm{RAND}}^{A_{0} A_{1} \rightarrow A^{\prime}} \equiv \sqrt{\left|A^{\prime \prime}{ }_{0}\right|\left|A^{\prime \prime}{ }_{1}\right|} \mathrm{op}^{A^{\prime \prime}{ }_{0} A^{\prime \prime}{ }_{1} \rightarrow A^{\prime}}\left(\Omega^{\prime}(\theta)\right)\left(U_{0}^{A^{\prime \prime}{ }_{0}} W_{0}^{A_{1} \rightarrow A^{\prime \prime}{ }_{0}} \otimes U_{1}^{A^{\prime \prime}{ }_{1}} W_{1}^{A_{1} \rightarrow A^{\prime \prime}{ }_{1}}\right)
$$

where $W_{0}$ and $W_{1}$ are isometric embeddings and $|\Omega(\theta)\rangle^{A^{\prime \prime}{ }_{0} A^{\prime \prime}{ }_{1} A^{\prime}}=U_{\theta}^{A^{\prime \prime} \rightarrow A^{\prime \prime}{ }_{0} A^{\prime \prime}}|\Omega\rangle^{A^{\prime \prime} A^{\prime}}$.

We will define two intermediary states, which will allow us to randomise over one input at a time, keeping the other fixed. To that end, define the states

$$
\begin{aligned}
& \left|\omega_{\text {Alice }_{0}}\left(U_{1}\right)\right\rangle^{A^{\prime \prime}{ }_{0} A^{\prime} B_{1} R_{1}}:=\sqrt{\left|A^{\prime \prime}{ }_{1}\right|}\left(\operatorname{op}^{A^{\prime \prime}{ }_{1} \rightarrow A^{\prime \prime}{ }_{0} A^{\prime}}\left(\Omega^{\prime}(\theta)\right) U_{1} W_{1}|\psi\rangle^{A_{1} B_{1} R_{1}}\right) \\
& \left|\omega_{\text {Alice }_{1}}\left(U_{0}\right)\right\rangle^{A^{\prime \prime}{ }_{1} A^{\prime} R_{0}}:=\sqrt{\left|A^{\prime \prime}{ }_{0}\right|}\left(\mathrm{op}^{A^{\prime \prime}{ }_{0} \rightarrow A^{\prime \prime}{ }_{1} A^{\prime}}\left(\Omega^{\prime}(\theta)\right) U_{0} W_{0}|\eta\rangle^{A_{0} R_{0}}\right)
\end{aligned}
$$

Also define

$$
\left|\omega_{\text {Alice }_{0}}\left(U_{1}\right)\right\rangle^{A^{\prime \prime}{ }_{0} B_{1} R_{1} C E}:=\mathcal{U}_{\mathcal{N}}^{A^{\prime} \rightarrow C E}\left|\omega_{\text {Alice }_{0}}\left(U_{1}\right)\right\rangle^{A^{\prime \prime}{ }_{0} A^{\prime} B_{1} R_{1}}
$$

and

$$
\left|\omega_{\text {Alice }_{1}}\left(U_{0}\right)\right\rangle^{A^{\prime \prime} R_{0} C E}:=\mathcal{U}_{\mathcal{N}}^{A^{\prime} \rightarrow C E}\left|\omega_{\text {Alice }_{1}}\left(U_{0}\right)\right\rangle^{A^{\prime \prime}{ }_{1} A^{\prime} R_{0}}
$$

The Decoupling Step : As promised, these states will help us randomise over one input, while holding the other fixed, as can be seen via two applications of the single sender decoupling theorem:

$$
\begin{aligned}
& \mathbb{E}_{U_{0}}\left[\|\left|A^{\prime \prime}{ }_{0}\right| \operatorname{Tr}_{C} \mathcal{U}_{\mathcal{N}} \text { op }^{A^{\prime \prime}{ }_{0} \rightarrow A^{\prime} B_{1} R_{1}}\left(\omega_{\text {Alice }_{0}}\left(U_{1}\right)\right) U_{0} W_{0} \cdot \eta^{R_{0} A_{0}}-\eta^{R_{0}} \otimes \omega_{\text {Alice }_{0}}^{B_{1} R_{1} E}\left(U_{1}\right) \|_{1}\right] \\
& \leq 2^{-\frac{1}{2} H_{2}^{\epsilon}\left(A_{0} \mid R_{0}\right)_{\eta}-\frac{1}{2} H_{\min }^{\epsilon}\left(A^{\prime \prime}{ }_{0} \mid B_{1} R_{1} E\right)_{\omega_{\text {Alice }}}\left(U_{1}\right)}+8 \epsilon \\
& \text { (dec_Alice }) \\
& \mathbb{E}_{U_{1}}\left[\|\left|A^{\prime \prime}{ }_{1}\right| \operatorname{Tr}_{C R_{0}} \mathcal{U}_{\mathcal{N}} \text { op }^{A^{\prime \prime}{ }_{1} \rightarrow A^{\prime} R_{0}}\left(\omega_{\text {Alice }_{1}}\left(U_{0}\right)\right) U_{1} W_{1} \cdot \psi^{R_{1} A_{1}}-\psi^{R_{1}} \otimes \omega_{\text {Alice }_{1}}^{E}\left(U_{0}\right) \|_{1}\right] \\
& \leq 2^{-\frac{1}{2} H_{2}^{\epsilon}\left(A_{1} \mid R_{1}\right)_{\psi}-\frac{1}{2} H_{\min }^{\epsilon}\left(A^{\prime \prime}{ }_{1} \mid E\right)_{\omega_{\text {Alice }_{1}}\left(U_{0}\right)}+8 \epsilon} \\
& \text { (dec_Alice } 1 \text { ) }
\end{aligned}
$$

Notice that in the second expression, we traces out both $C$ and $R_{0}$. This is in anticipation of the fact that Alice 1 will use the correlations in $R_{0}$ to decode Alice ${ }_{2}$ 's input. 
The inequalities above will go towards proving the the existence of a good decoder. To show that a good encoder exists, we use the single sender decoupling theorem twice more :

$$
\begin{aligned}
& \mathbb{E}_{U_{0}}\left[\|\left|A^{\prime \prime}{ }_{0}\right| \operatorname{Tr}_{A^{\prime}} \text { op }^{A^{\prime \prime}{ }_{0} \rightarrow A^{\prime} B_{1} R_{1}}\left(\omega_{\text {Alice }_{0}}\left(U_{1}\right)\right) U_{0} W_{0} \cdot \eta^{R_{0} A_{0}}-\eta^{R_{0}} \otimes \omega_{\text {Alice }_{0}}^{B_{1} R_{1}}\left(U_{1}\right) \|_{1}\right] \\
& \leq 2^{\frac{1}{2} H_{\max }^{\epsilon}\left(A_{0}\right)_{\eta}-\frac{1}{2} H_{\min }^{\epsilon}\left(A^{\prime \prime}{ }_{0} \mid B_{1} R_{1}\right)_{\omega_{\text {Alice }_{0}}}\left(U_{1}\right)}+8 \epsilon \\
& \text { (enc_Alice } 0 \text { ) } \\
& \mathbb{E}_{U_{1}}\left[\|\left|A^{\prime \prime}{ }_{1}\right| \operatorname{Tr}_{A^{\prime}} \text { op }^{A^{\prime \prime}{ }_{1} \rightarrow A^{\prime \prime}{ }_{0} A^{\prime}}\left(\Omega^{\prime}(\theta)\right) U_{1} W_{1} \cdot \psi^{R_{1} B_{1} A_{1}}-\psi^{R_{1} B_{1}} \|_{1}\right] \\
& \leq 2^{\frac{1}{2} H_{\text {max }}^{\epsilon}\left(A_{1}\right)_{\psi}-\frac{1}{2} H_{\min }^{\epsilon}\left(A^{\prime \prime}\right)_{\Omega^{\prime}(\theta)}+8 \epsilon} \\
& \text { (enc_Alice } 1 \text { ) }
\end{aligned}
$$

First, note that by Theorem 2.6, the following holds:

$$
\begin{aligned}
\mathcal{E}_{\mathrm{RAND}}^{A_{0} A_{1} \rightarrow A^{\prime}}|\eta\rangle^{A R_{0}}|\psi\rangle^{B B_{1} R_{1}} & =\sqrt{\left|A^{\prime \prime}{ }_{0}\right|} \text { op }^{A^{\prime \prime}{ }_{0} \rightarrow A^{\prime} B_{1} R_{1}}\left(\omega_{\text {Alice }_{0}}\left(U_{1}\right)\right) U_{0} W_{0}|\eta\rangle^{R_{0} A_{0}} \\
& =\sqrt{\left|A^{\prime \prime}{ }_{1}\right|} \text { op }^{A^{\prime} R_{0}}\left(\omega_{\text {Alice }_{1}}\left(U_{0}\right)\right) U_{1} W_{1}|\psi\rangle^{A_{1} R_{1} B_{1}} \\
& :=\mid \text { GLOBAL }\rangle_{0}^{A_{0} A^{\prime} R_{0} R_{1}}
\end{aligned}
$$

Also, we observe that the first term in the LHS of Eq. (enc_Alice 1 ) is, by definition, equal to $\omega_{\text {Alice }_{0}}^{B_{1} R_{1}}\left(U_{1}\right)$.

Next, we need to derandomise the four inequalities, i.e. we will show that there exist fixed $U_{0}$ and $U_{1}$ such that all four inequalities hold. The issue is that the RHS of each inequality, aside from Eq. (enc_Alice ${ }_{1}$ ), has min entropy terms which are functions of $\omega_{\text {Alice }_{0}}\left(U_{1}\right)$ and $\omega_{\text {Alice }}\left(U_{2}\right)$, when we want bounds in terms of the control state $\Omega^{\prime}(\theta)$. For example, consider the expression $H_{\min }^{\epsilon}\left(A^{\prime \prime}{ }_{0} \mid B_{1} R_{1} E\right)_{\omega_{\text {Alice }_{0}}\left(U_{1}\right)}$. To get the correct upper bounds, we would have to prove an inequality of the following kind:

$$
H_{\min }^{\epsilon}\left(A^{\prime \prime}{ }_{0} \mid B_{1} R_{1} E\right)_{\omega_{\text {Alice }_{0}}\left(U_{1}\right)} \geq H_{\min }^{\epsilon}\left(A^{\prime \prime}{ }_{0} \mid A^{\prime \prime}{ }_{1} E\right)_{\Omega^{\prime}(\theta)}
$$

for every $U_{1}$. Unfortunately this is not true. The way we get around this issue is that, we show that on average over the choice of $U_{1}$, the following inequality holds true

$$
H_{\min }^{\epsilon}\left(A^{\prime \prime}{ }_{0} \mid B_{1} R_{1} E\right)_{\omega_{\text {Alice }_{0}}\left(U_{1}\right)} \geq H_{\min }^{O\left(\epsilon^{2}\right)}\left(A^{\prime \prime}{ }_{0} \mid A^{\prime \prime}{ }_{1} E\right)_{\Omega^{\prime}(\theta)}-O(1)
$$

(average_dpi)

The way we prove the above statement is by via the almost CPTP maps defined in Section 2.6, specifically, Theorem 2.18. The state of Theorem 2.18, adapted to the current setting, implies that, there exist with constant probability, two fixed unitaries $U_{0}$ and $U_{1}$ such that Eq. (dec_Alice 0 ) and Eq. (average_dpi) hold simultaneously.

To show that a similar results holds for Eq. (dec_Alice 1 ) and Eq. (enc_Alice 0 ), note that the proof of Theorem 2.18 requires a union bound over 3 bad events, each with probability at most $\frac{1}{k}$, where $k$ is some adjustable integer parameter $\geq 4$. In this case, we will need to apply Theorem 2.18 to Eq. (dec_Alice 1 ), Eq. (dec_Alice $)$ and Eq. (enc_Alice 1 ), each of which will contribute 3 bad events. Additionally, we will also need to show that, for our fixed $U_{0}$ and $U_{1}$, Eq. (enc_Alice 1 ) 
holds. Thus, we have to take a union bound over 10 bad events. Thus, a good choice for the adjustable parameter $k$ is 20 .

Set $\epsilon_{0}:=\frac{\epsilon^{2}}{800}$. The above discussion then implies that there exist fixed unitaries $U_{0}$ and $U_{1}$ such that Eq. (dec_Alice 0 ), Eq. (dec_Alice 1 ), Eq. (enc_Alice $)$ and Eq. (enc_Alice 1 ) can be written as:

$$
\begin{aligned}
& \left\|\operatorname{Tr}_{C} \mathcal{U}_{\mathcal{N}} \cdot \mathrm{GLOBAL}_{0}-\eta^{R_{0}} \otimes \omega_{\text {Alice }_{0}}^{B_{1} R_{1} E}\left(U_{1}\right)\right\|_{1} \\
& \leq 20 \cdot 2^{-\frac{1}{2} H_{2}^{\epsilon}\left(A_{0} \mid R_{0}\right)_{\eta}-\frac{1}{2} H_{\min }^{\varepsilon_{0}}\left(A^{\prime \prime}{ }_{0} \mid A^{\prime \prime}{ }_{1} E\right)_{\mathcal{U}_{\mathcal{N}} \Omega^{\prime}(\theta)}}+160 \epsilon \\
& :=\delta_{\mathrm{dec}}(0) \\
& \| \operatorname{Tr}_{C R_{0}} \mathcal{U}_{\mathcal{N}} \cdot \operatorname{Tr}_{B_{1}} \text { GLOBAL }_{0}-\psi^{R_{1}} \otimes \omega_{\text {Alice }_{1}}^{E}\left(U_{0}\right) \|_{1} \\
& \leq 20 \cdot 2^{-\frac{1}{2} H_{2}^{\epsilon}\left(A_{1} \mid R_{1}\right)_{\psi}-\frac{1}{2} H_{\min }^{\epsilon_{0}}\left(A^{\prime \prime} 1 \mid E\right)_{\Omega^{\prime}(\theta)}+160 \epsilon} \\
& :=\delta_{\operatorname{dec}}(1) \\
& \left\|\operatorname{Tr}_{A^{\prime}} \mathrm{GLOBAL}_{0}-\eta^{R_{0}} \otimes \psi^{R_{1} B_{1}}\right\|_{1} \\
& \leq 20 \cdot 2^{\frac{1}{2} H_{\max }^{\epsilon}\left(A_{0}\right)_{\eta}-\frac{1}{2} H_{\min }^{\epsilon_{0}}\left(A^{\prime \prime}{ }_{0} \mid A^{\prime \prime}{ }_{1}\right)_{\Omega^{\prime}(\theta)}+160 \epsilon}
\end{aligned}
$$

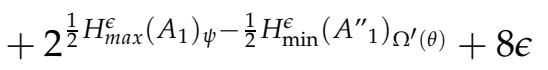

$$
\begin{aligned}
& :=\delta_{\text {enc }}(0)+\delta_{\text {enc }}(1) \\
& :=\delta_{\mathrm{enc}}
\end{aligned}
$$

where we have use the triangle inequality on the derandomised versions of Eq. (dec_Alice 0 ) and Eq. $\left(\right.$ dec_Alice $\left._{1}\right)$ to get the last inequality. One technical issue is that since $\mathcal{E}_{\text {RAND }}$ is not a TP map, we cannot apply Uhlmann's theorem directly, since $\mid$ GLOBAL $\rangle_{0}$ may not be a unit vector. To circumvent this we use Theorem 2.13. Suppose $c_{0}:=\frac{1}{\operatorname{Tr}\left[\mathrm{GLBAL}_{0}\right]}$. Then, by Theorem 2.13,

$$
\left\|c_{0} \operatorname{Tr}_{C} \mathcal{U}_{\mathcal{N}} \cdot \mathrm{GLOBAL}_{0}-\eta^{R_{0}} \otimes \omega_{\text {Alice }_{1}}^{B_{1} R_{1} E}\left(U_{1}\right)\right\|_{1} \leq 2 \delta_{\mathrm{dec}}(0)
$$

We can make similar statements for the other two inequalities.

The Successive Cancellation Step: Roughly, the idea is as follows : Bob first decodes for Alice $_{0}$ 's input, and recovers a state close to $|\eta\rangle^{A_{0} R_{0}}$. That such an isometric decoder exists is inferred from the normalised version of decoding condition for Alice ${ }_{0}$ and Uhlmann's theorem. After this step, Bob possesses the registers $A_{0}$ and $F_{1}$, where $F_{1}$ contains correlations with the rest of the parties involved in the protocol.

Bob keeps the $A_{0}$ system aside, and brings in the locally prepared state $|\eta\rangle^{\stackrel{\circ}{0}_{0} \stackrel{\circ}{R}_{0}}$. He then inverts the decoding procedure on the systems $\AA_{0} F_{1}$ and maps to a state which is close to GLOBAL $\mathrm{L}_{0}$, with the important difference that now the register $\stackrel{\circ}{R}_{0}$ is in Bob's possession. 
Finally, from from the decoding condition for Alice ${ }_{1}$ and Uhlmann's theorem, we infer the existence of a decoder which uses not only the registers $B B_{1}$ for decoding but also utilises the correlations that $\stackrel{\circ}{R}_{0}$ has with $R_{1}$. This allows Alice 1 to transmit at a higher rate than the simple point to point case.

To make things precise, we first define the states $\left|\Omega^{\prime}(\theta)\right\rangle^{A^{\prime \prime}{ }_{0} A^{\prime \prime}}{ }_{1} A^{\prime}$ and $|\eta\rangle^{\stackrel{\circ}{A_{0} R_{0}}}$, which are all copies of the corresponding states defined earlier, but with the registers $A_{0}, A^{\prime \prime}{ }_{0}$ and $R_{0}$ replaced by $\AA_{0}, \stackrel{\circ}{A}^{\prime \prime}{ }_{0}$ and $\stackrel{\circ}{R}_{0}$, which are all local to Bob. Following this convention, we can also define the state $\left|\omega_{\text {Alice }_{0}}\left(U_{1}\right)\right\rangle^{A^{\prime \prime}{ }_{0} A^{\prime} B_{1} R_{1}}$ analogously. Next, define

$$
\mid \text { TARGET }\rangle^{\stackrel{\circ}{R_{0}} R_{1} B_{1} A^{\prime}}:=\sqrt{\left|A^{\prime \prime}{ }_{0}\right|} \text { op }^{\stackrel{\circ}{A^{\prime \prime}}{ }_{0} \rightarrow A^{\prime} B_{1} R_{1}}\left(\omega_{\text {Alice }_{0}}\left(U_{1}\right)\right)\left(U_{0} W_{0}|\eta\rangle^{\stackrel{\circ}{0}_{0} \stackrel{\circ}{R}_{0}}\right)
$$

where the unitary $U_{0}$ acts on the register $\AA^{\prime \prime}{ }_{0}$, and $W_{0}$ maps $\AA_{0} \rightarrow \AA^{\prime \prime}{ }_{0}$.

By the decoding condition for Alice ${ }_{0}$ and Uhlmann's theorem, we see that there exists an isometric decoder $V_{\text {Alice }}^{B \rightarrow A_{0} F_{1}}$ such that

$$
\left\|c_{0} \cdot V_{\text {Alice }_{0}} \mathcal{U}_{\mathcal{N}} \cdot \mathrm{GLOBAL}_{0}-\eta^{A_{0} R_{0}} \zeta_{1} F_{1} R_{1} B_{1} E\right\|_{1} \leq 2 \sqrt{2 \delta_{\mathrm{dec}}(0)}
$$

where $\eta$ and $\zeta_{1}$ are pure and $\zeta_{1}$ is a purification of $\omega_{\text {Alice }_{0}}\left(U_{1}\right)^{B_{1} R_{1} E}$.

Since GLOBAL $L_{0}$ and TARGET are the same states aside from renaming the $R_{0}$ register, the above result also implies that there exists an isometry $V_{\text {Sim_Alice }_{0}}^{B \rightarrow \stackrel{\AA}{A}_{0} F_{1}}$ such that

$$
\| c_{0} \cdot V_{\text {Sim_Alice }_{0}} \mathcal{U}_{\mathcal{N}} \cdot \text { TARGET }-\eta^{\stackrel{\circ}{A}_{0} \stackrel{\circ}{R}_{0}} \zeta_{1} F_{1} R_{1} B_{1} E \|_{1} \leq 2 \sqrt{2 \delta_{\text {dec }}(0)}
$$

Simple algebra and a triangle inequality then implies that

$$
c_{0} \cdot \| V_{\text {Sim }_{-} \text {Alice }} \eta \eta^{\stackrel{\circ}{\AA_{0} R_{0}}} V_{\text {Alice }_{1}} \mathcal{U}_{\mathcal{N}} \cdot \text { GLOBAL }_{0}-\eta^{A_{0} R_{0}} \mathcal{U}_{\mathcal{N}} \text { TARGET } \|_{1} \leq 4 \sqrt{2 \delta_{\text {dec }}(0)}
$$

Note that, with the registers $A^{\prime \prime}{ }_{0}$ and $R_{0}$ replaced by $\stackrel{\circ}{\prime \prime}_{0}$ and $\stackrel{\circ}{R}_{0}$, and making the appropriate changes in the definition of the state $\omega_{\text {Alice }_{0}}\left(U_{0}\right)$, the decoding condition for Alice ${ }_{1}$ along with Uhlmann's theorem implies that, there exists a decoding isometry $V_{\mathrm{Alice}}^{B B_{1} \stackrel{\circ}{R}_{0} \rightarrow A_{1} B_{1} F_{2}}$ such that

$$
\| c_{0} \cdot V_{\text {Alice }_{1}} \mathcal{U}_{\mathcal{N}} \cdot \text { TARGET }-\psi^{A_{1} R_{1} B_{1}} \zeta_{2}{ }_{2} E \|_{1} \leq 2 \sqrt{2 \delta_{\mathrm{dec}}(1)}
$$

Defining $V_{\text {Bob }}^{B B_{1} \stackrel{\circ}{R}_{0} \AA_{0}} \rightarrow A_{0} A_{1} B_{1} F_{2}:=V_{\text {Alice }_{1}} \circ V_{\text {Sim_Alice }_{0}}^{-1} \circ V_{\text {Alice }_{0}}$ and collating the above arguments, we see that

$$
\left\|c_{0} \cdot V_{\mathrm{Bob}} \mathcal{U}_{\mathcal{N}} \cdot \eta^{\stackrel{\circ}{0}_{0} \stackrel{\circ}{0}_{0}} \mathrm{GLOBAL}_{0}-\eta^{A_{0} R_{0}} \psi^{A_{1} R_{1} B_{1}} \zeta_{2} F_{2} E\right\|_{1} \leq 4 \sqrt{2 \delta_{\mathrm{dec}}(0)}+2 \sqrt{2 \delta_{\mathrm{dec}}(1)}
$$

Isometric Encoding: We finally show that there exists an isometric encoder. To do this, we simply consider the decoupling condition

$$
\left\|c_{0} \cdot \operatorname{Tr}_{A^{\prime}} \mathrm{GLOBAL}_{0}-\eta^{R_{0}} \otimes \psi^{R_{1} B_{1}}\right\|_{1} \leq 2 \delta_{\mathrm{enc}}
$$


Applying Uhlmann's theorem implies that, there exists an encoding isometry $V_{\text {enc }}^{A_{0} A_{1} \rightarrow A^{\prime}}$ such that

$$
\left\|c_{0} \cdot \mathrm{GLOBAL}_{0}-V_{\mathrm{enc}} \eta^{A_{0} R_{0}} \otimes \psi^{A_{1} R_{1} B_{1}}\right\|_{1} \leq 2 \sqrt{2 \delta_{\text {enc }}}
$$

Tracing out $F_{2} E$ and another triangle inequality then shows that the proposition is true.

\section{The Multiple Access Channel}

We now state our one-shot inner bounds for the QMAC with limited entanglement assistance. But first we need a technical proposition akin to Theorem 3.4, from which our inner bounds follow easily.

Proposition 4.1 Consider the quantum multiple access channel $\mathcal{N}^{A^{\prime} B^{\prime} \rightarrow C}$. Consider a pure 'control state' $|\sigma\rangle^{A^{\prime \prime} B^{\prime \prime} A^{\prime} B^{\prime}}:=|\Omega\rangle^{A^{\prime \prime} A^{\prime}}|\Delta\rangle^{B^{\prime \prime} B^{\prime}}$. Let $|\psi\rangle^{A_{1} C_{1} R_{1}} \otimes|\eta\rangle^{A_{0} C_{0} R_{0}}$ and $|\phi\rangle^{B D S}$ be the states that are to be sent to Charlie through the channel by Alice and Bob respectively, where $C_{0}, C_{1}, D$ model the side information about the respective messages $A_{0}, A_{1}, B$ that Charlie possesses and $R_{0}, R_{1}, S$ are reference systems that are untouched by channel and coding operators. Let II denote the identity superoperator. For $\theta \in[0,1]$, let $U_{\theta}^{A^{\prime \prime}}$ be a splitting scheme. We define $|\sigma(\theta)\rangle^{A_{0}^{\prime \prime} A_{1}^{\prime \prime} A^{\prime} B^{\prime \prime} B^{\prime}}:=U_{\theta}|\Omega\rangle^{A^{\prime \prime} A^{\prime}}|\Delta\rangle^{B^{\prime \prime} B^{\prime}}$ and

$$
\sigma(\theta)^{A_{0}^{\prime \prime} A_{1}^{\prime \prime} B^{\prime \prime} C}:=\left(\mathcal{N}^{A^{\prime} B^{\prime} \rightarrow C} \otimes \mathbb{I}^{A_{0}^{\prime \prime} A_{1}^{\prime \prime} B^{\prime \prime}}\right)\left(\sigma(\theta)^{A_{0}^{\prime \prime} A_{1}^{\prime \prime} A^{\prime} B^{\prime \prime} B^{\prime}}\right) .
$$

Then there exist encoding maps $\mathcal{A}^{A_{0} A_{1} \rightarrow A^{\prime}}, \mathcal{B}^{B \rightarrow B^{\prime}}$ and a decoding map $\mathcal{C}^{C C_{0} C_{1} D \rightarrow A_{0} C_{0} A_{1} C_{1} B D}$ such that

$$
\begin{aligned}
& \|\left(\mathcal{C} \otimes \mathbb{I}^{R_{0} R_{1} S}\right)\left(\left(\mathcal{N} \otimes \mathbb{I}^{C_{0} R_{0} C_{1} R_{1} D S}\right)(\right. \\
& \left.\left.\left(\mathcal{A} \otimes \mathcal{B} \otimes \mathbb{I}^{C_{0} R_{0} C_{1} R_{1} D S}\right)((\eta \otimes \psi) \otimes \phi)\right)\right) \\
& \quad-\eta \otimes \psi \otimes \phi \|_{1} \leq \delta
\end{aligned}
$$

where $\delta:=\delta_{\mathrm{enc}}+\delta_{\mathrm{dec}}$ and

$$
\begin{aligned}
& \delta_{\text {enc }}:=4\left(\sqrt{\min \left\{\delta_{\text {enc }}(0,1), \delta_{\text {enc }}(1,0)\right\}}+\sqrt{\delta_{\text {enc }}(2)}\right) \\
& \delta_{\mathrm{dec}}:=8\left(\sqrt{\delta_{\mathrm{dec}}(0)}+\sqrt{\delta_{\mathrm{dec}}(1)}+\sqrt{\delta_{\mathrm{dec}}(2)}\right) \\
& \delta_{\text {enc }}(0,1)=20 \cdot 2^{\frac{1}{2}\left(H_{\max }^{\epsilon}\left(A_{0}\right)_{\eta}-H_{\min }^{\frac{\epsilon^{2}}{800}}\left(A_{0}^{\prime \prime} \mid A_{1}^{\prime \prime}\right)_{\sigma(\theta)}\right)} \\
& +20 \cdot 2^{\frac{1}{2}\left(H_{\max }^{\epsilon}\left(A_{1}\right)_{\psi}-H_{2}^{\epsilon}\left(A_{1}^{\prime \prime}\right)_{\sigma(\theta)}\right)}+320 \epsilon, \\
& \delta_{\text {enc }}(1,0)=20 \cdot 2^{\frac{1}{2}\left(H_{\max }^{\epsilon}\left(A_{1}\right)_{\eta}-H_{\min }^{\frac{\epsilon^{2}}{800}}\left(A_{1}^{\prime \prime} \mid A_{0}^{\prime \prime}\right)_{\sigma(\theta)}\right)} \\
& +20 \cdot 2^{\frac{1}{2}\left(H_{\max }^{\epsilon}\left(A_{0}\right)_{\psi}-H_{2}^{\epsilon}\left(A_{0}^{\prime \prime}\right)_{\sigma(\theta)}\right)}+320 \epsilon, \\
& \delta_{\mathrm{enc}}(2)=20 \cdot 2^{\frac{1}{2}\left(H_{\max }^{\epsilon}(B)_{\phi}-H_{2}^{\epsilon}\left(B^{\prime \prime}\right)_{\sigma(\theta)}\right)}+160 \epsilon \text {, } \\
& \delta_{\mathrm{dec}}(0):=20 \cdot 2^{-\frac{1}{2}\left(H_{2}^{\epsilon}\left(A_{0} \mid R_{0}\right)_{\eta}+I_{\min }^{\frac{\epsilon^{2}}{800}}\left(A_{0}^{\prime \prime}>C\right)_{\sigma(\theta)}\right)}+160 \epsilon \text {, } \\
& \delta_{\operatorname{dec}}(1):=20 \cdot 2^{-\frac{1}{2}\left(H_{2}^{\epsilon}\left(A_{1} \mid R_{1}\right)_{\psi}+I_{\min }^{\frac{\epsilon^{2}}{800}}\left(A_{1}^{\prime \prime}>C A_{0}^{\prime \prime} B^{\prime \prime}\right)_{\sigma(\theta)}\right)}+160 \epsilon \text {, } \\
& \delta_{\mathrm{dec}}(2):=20 \cdot 2^{-\frac{1}{2}\left(H_{2}^{\epsilon}(B \mid S)_{\phi}+I_{\min }^{\frac{\epsilon^{2}}{800}}\left(B^{\prime \prime}>C A_{0}^{\prime \prime}\right)_{\sigma(\theta)}\right)}+160 \epsilon .
\end{aligned}
$$


Theorem 4.1 immediately implies the following theorem, by the arguments presented in Theorem 3.5.

Theorem 4.2 Consider the setting of Proposition 4.1 Let $Q_{A}, E_{A}, Q_{B}, E_{B}$ be the number of message qubits and number of available ebits of Alice and Bob respectively. Let $\theta, \epsilon \in[0,1]$ and $\epsilon_{0}:=\frac{\epsilon^{2}}{800}$. Then there exist encoding and decoding maps such that any message cum ebit rate 4-tuple satisfying either the following set of constraints or the set obtained by interchanging $A_{0}^{\prime \prime}$ with $A_{1}^{\prime \prime}, Q_{A}(0)$ with $Q_{A}(1)$ and $E_{A}(0)$ with $E_{A}(1)$ in the right hand sides of the first two inequalities, is achievable with error at most $100 \sqrt{\epsilon}$ :

$$
\begin{aligned}
Q_{A} & =Q_{A}(0)+Q_{A}(1), E_{A}=E_{A}(0)+E_{A}(1), \\
Q_{A}(0)+E_{A}(0) & <H_{\min }^{\epsilon_{0}}\left(A_{0}^{\prime \prime} \mid A_{1}^{\prime \prime}\right)_{\sigma(\theta)}+4 \log \epsilon, \\
Q_{A}(1)+E_{A}(1) & <H_{2}^{\epsilon}\left(A_{1}^{\prime \prime}\right)_{\sigma(\theta)}+4 \log \epsilon, \\
Q_{A}(0)-E_{A}(0) & <I_{\min }^{\epsilon_{0}}\left(A_{0}^{\prime \prime}>C\right)_{\sigma(\theta)}+4 \log \epsilon, \\
Q_{A}(1)-E_{A}(1) & <I_{\min }^{\epsilon_{0}}\left(A_{1}^{\prime \prime}>C A_{0}^{\prime \prime} B^{\prime \prime}\right)_{\sigma(\theta)}+4 \log \epsilon, \\
Q_{B}+E_{B} & <H_{2}^{\epsilon}\left(B^{\prime \prime}\right)_{\sigma(\theta)}+4 \log \epsilon, \\
Q_{B}-E_{B} & <I_{\min }^{\epsilon_{0}}\left(B^{\prime \prime}>C A_{0}^{\prime \prime}\right)_{\sigma(\theta)}+4 \log \epsilon .
\end{aligned}
$$

Proof:[Proof of Theorem 4.1] Preprocessing : Let $W_{0}^{A_{0} \rightarrow A^{\prime \prime}{ }_{0}}, W_{1}^{A_{1} \rightarrow A^{\prime \prime}{ }_{1}}$ and $W_{2}^{A_{2}}$ be isometric embeddings such that

$$
\begin{aligned}
|\tilde{\eta}\rangle & :=W_{0}^{A_{0} \rightarrow A^{\prime \prime}}{ }_{0}|\eta\rangle \\
|\tilde{\psi}\rangle: & :=W_{1}^{A_{1} \rightarrow A^{\prime \prime}{ }_{1}}|\psi\rangle \\
|\tilde{\varphi}\rangle & :=W_{2}^{A_{2} \rightarrow A^{\prime \prime}{ }_{2}}|\varphi\rangle
\end{aligned}
$$

Global State: We define

$$
\begin{aligned}
& \left|A^{\prime \prime}{ }_{0} A^{\prime \prime}{ }_{1} B^{\prime \prime}\right| \operatorname{op}^{A{ }^{\prime \prime} A^{\prime \prime}{ }_{1} B^{\prime \prime} \rightarrow A^{\prime} B^{\prime}}(\sigma(\theta)) \cdot\left(U_{0} \otimes U_{1} \otimes U_{2}\right) \cdot\left(\tilde{\eta}^{R_{0} C_{0} A^{\prime \prime}{ }_{0}} \otimes \tilde{\psi}^{R_{1} A^{\prime \prime}{ }_{1} C_{1}} \otimes \tilde{\varphi}^{S B^{\prime \prime} D}\right) \\
& :=\mathrm{GLOBAL}_{0}
\end{aligned}
$$

\section{Intermediary States :}

Encoding

$$
\left|\omega_{\text {enc }}\left(U_{1}\right)\right\rangle^{A_{0}{ }^{\prime \prime} C_{1} R_{1} A^{\prime}}:=\sqrt{\left|A^{\prime \prime}{ }_{1}\right|}\left(\operatorname{op}^{A^{\prime \prime}{ }_{1} \rightarrow A_{0}{ }^{\prime \prime} A^{\prime}}\left(\Omega^{\prime}(\theta)\right) U_{1}^{A^{\prime \prime}{ }_{1}}|\tilde{\psi}\rangle^{A_{1}{ }^{\prime \prime} C_{1} R_{1}}\right)
$$

Decoding

$$
\begin{aligned}
& \left|\omega_{\text {Alice }_{0}}\left(U_{1}, U_{2}\right)\right\rangle^{A^{\prime \prime}{ }_{0} A^{\prime} B^{\prime} C_{1} R_{1} D S}:=\sqrt{\left|A^{\prime \prime}{ }_{1} B^{\prime \prime}\right|}\left(\operatorname{op}^{A^{\prime \prime}{ }_{1} B^{\prime \prime} \rightarrow A^{\prime \prime}{ }_{0} A^{\prime} B^{\prime}}(\sigma(\theta))\left(U_{1}^{A^{\prime \prime}}{ }_{1} \otimes U_{2}^{B^{\prime \prime}}\right)|\tilde{\psi}\rangle^{A^{\prime \prime}{ }_{1} C_{1} R_{1}}|\tilde{\varphi}\rangle^{B^{\prime \prime} D S}\right) \\
& \left|\omega_{\text {Bob }}\left(U_{0}, U_{1}\right)\right\rangle^{B^{\prime \prime} A^{\prime} B^{\prime} C_{1} R_{1} C_{0} R_{0}}:=\sqrt{\left|A^{\prime \prime}{ }_{0} A^{\prime \prime}{ }_{1}\right|}\left(\operatorname{op}^{A^{\prime \prime}{ }_{0} A^{\prime \prime}{ }_{1} \rightarrow B^{\prime \prime} A^{\prime} B^{\prime}}(\sigma(\theta))\left(U_{0}^{A^{\prime \prime}{ }_{0}} \otimes U_{1}^{A^{\prime \prime}{ }_{1}}\right)|\tilde{\eta}\rangle^{A^{\prime \prime}{ }_{0} C_{0} R_{0}}|\tilde{\psi}\rangle^{A^{\prime \prime}{ }_{1} C_{1} R_{1}}\right) \\
& \left|\omega_{\text {Alice }_{1}}\left(U_{0}, U_{2}\right)\right\rangle^{A^{\prime \prime}{ }_{1} A^{\prime} B^{\prime} C_{0} R_{0} S D}:=\sqrt{\left|A^{\prime \prime}{ }_{0} B^{\prime \prime}\right|}\left(\operatorname{op}^{A^{\prime \prime}{ }_{0} B^{\prime \prime} \rightarrow A^{\prime \prime}{ }_{1} A^{\prime} B^{\prime}}(\sigma(\theta))\left(U_{0}^{A^{\prime \prime}{ }_{0}} \otimes U_{2}^{B^{\prime \prime}}\right)|\tilde{\eta}\rangle^{A^{\prime \prime}{ }_{0} C_{0} R_{0}}|\tilde{\varphi}\rangle^{B^{\prime \prime} D S}\right)
\end{aligned}
$$


The Decoupling Step : We will apply Theorem 2.18 simultaneously to all 4 intermediate states. We will have to choose an appropriate value of the constant $k$ to make the derandomisation work. To that end, notice that each application of Theorem 2.18 has a union bound over 3 bad events. Generalising over all four intermediate states implies that we will have to take a union bound over 12 bad events. In addition, we have to include two additional bad events in the union bound that originate from applying the single sender decoupling theorem to show that good encoders exist. Thus, setting $k=20$ we see that there exist fixed $U_{0}, U_{1}$ and $U_{2}$ such that the following conditions are satisfied simultaneously:

$$
\begin{aligned}
& \|\left|A^{\prime \prime}{ }_{0}\right| \operatorname{Tr}_{C} \mathcal{U}_{\mathcal{N}}^{A^{\prime} B^{\prime} \rightarrow C E}\left(\text { op }^{A^{\prime \prime}{ }_{0} \rightarrow A^{\prime} B^{\prime} C_{1} R_{1} D S}\left(\omega_{\text {Alice }_{0}}\left(U_{1}, U_{2}\right)\right) \cdot\left(U_{0} \cdot \tilde{\eta}^{A^{\prime \prime}{ }_{0} R_{0}}\right)\right) \\
& -\eta^{R_{0}} \otimes \omega_{\text {Alice }_{0}}\left(U_{1}, U_{2}\right)^{C_{1} R_{1} D S E} \|_{1} \\
& \leq 20 \cdot 2^{-\frac{1}{2} H_{2}^{\epsilon}\left(A_{0} \mid R_{0}\right)_{\eta}-\frac{1}{2} H_{\min }^{\frac{\epsilon^{2}}{800}}\left(A^{\prime \prime}{ }_{0} \mid A^{\prime \prime}{ }_{1} B^{\prime \prime} E\right)_{\sigma(\theta)}}+160 \epsilon \\
& :=\delta_{\operatorname{dec}}(0) \\
& \|\left|B^{\prime \prime}\right| \operatorname{Tr}_{C R_{0} C_{0}} \mathcal{U}_{\mathcal{N}}^{A^{\prime} B^{\prime} \rightarrow C E}\left(\operatorname{op}^{B^{\prime \prime} \rightarrow A^{\prime} B^{\prime} C_{1} R_{1} C_{0} R_{0}}\left(\omega_{\mathrm{Bob}}\left(U_{0}, U_{1}\right)\right) \cdot\left(U_{2} \cdot \tilde{\varphi}^{A^{\prime \prime}{ }_{2} S}\right)\right) \\
& -\varphi^{S} \otimes \omega_{\mathrm{Bob}}\left(U_{0}, U_{1}\right)^{C_{1} E R_{1}} \|_{1} \\
& \leq 20 \cdot 2^{-\frac{1}{2} H_{2}^{\epsilon}(B \mid S)_{\varphi}-\frac{1}{2} H_{\min }^{\frac{\epsilon^{2}}{800}}\left(B^{\prime \prime} \mid A^{\prime \prime}{ }_{1} E\right)_{\sigma(\theta)}}+160 \epsilon \\
& :=\delta_{\mathrm{dec}}(1) \\
& \|\left|A^{\prime \prime}{ }_{1}\right| \operatorname{Tr}_{C R_{0} C_{0} S D} \mathcal{U}_{\mathcal{N}}^{A^{\prime} B^{\prime} \rightarrow C E}\left(\operatorname{op}^{A^{\prime \prime}{ }_{1} \rightarrow A^{\prime} B^{\prime} C_{0} R_{0} S D}\left(\omega_{\text {Alice }_{1}}\left(U_{0}, U_{2}\right)\right) \cdot\left(U_{1} \cdot \tilde{\psi}^{R_{1} A^{\prime \prime}{ }_{1}}\right)\right) \\
& -\psi^{R_{1}} \otimes \omega_{\text {Alice }_{1}}\left(U_{0}, U_{2}\right)^{E} \|_{1} \\
& \leq 20 \cdot 2^{-\frac{1}{2} H_{\min }^{\frac{\epsilon^{2}}{800}}}\left(A^{\prime \prime}{ }_{1} \mid E\right)_{\sigma(\theta)}-\frac{1}{2} H_{2}^{\epsilon}\left(A_{1} \mid R_{1}\right)_{\psi}+160 \epsilon \\
& :=\delta_{\operatorname{dec}}(2) \\
& \left\|\left|A_{0}{ }^{\prime \prime}\right| \operatorname{Tr}_{A^{\prime}}\left(\mathrm{op}^{A_{0}{ }^{\prime \prime} \rightarrow C_{1} R_{1} A^{\prime}}\left(\omega_{\mathrm{enc}}\left(U_{1}\right)\right) \cdot\left(U_{0} \cdot \tilde{\eta}^{R_{0} C_{0} A^{\prime \prime}{ }_{0}}\right)\right)-\eta^{\mathrm{C}_{0} R_{0}} \otimes \omega_{\mathrm{enc}}\left(U_{1}\right)^{C_{1} R_{1}}\right\|_{1} \\
& \leq 20 \cdot 2^{\frac{1}{2} H_{\max }^{\epsilon}\left(A_{0}\right)_{\eta}-\frac{1}{2} H_{\min }^{\frac{\epsilon^{2}}{800}}\left(A^{\prime \prime}{ }_{0} \mid A^{\prime \prime}{ }_{1}\right)_{\sigma(\theta)}+160 \epsilon} \\
& :=\delta_{\text {enc }}(0) \\
& \left\|\left|A^{\prime \prime}{ }_{1}\right| \operatorname{Tr}_{A^{\prime \prime}{ }_{0} A^{\prime}}\left(\operatorname{op}^{A^{\prime \prime}{ }_{1} \rightarrow A^{\prime \prime}{ }_{0} A^{\prime}}\left(\Omega^{\prime}(\theta)\right)\left(U_{1} \cdot \tilde{\psi}^{A^{\prime \prime}{ }_{1} C_{1} R_{1}}\right)\right)-\psi^{C_{1} R_{1}}\right\|_{1} \\
& =\left\|\omega_{\text {enc }}\left(U_{1}\right)^{C_{1} R_{1}}-\psi^{C_{1} R_{1}}\right\|_{1} \\
& \leq 20 \cdot 2^{-\frac{1}{2} H_{\min }^{\epsilon}\left(A^{\prime \prime}\right)_{\sigma(\theta)}+\frac{1}{2} H_{\max }^{\epsilon}\left(A_{1}\right)_{\psi}}+160 \epsilon \\
& :=\delta_{\mathrm{enc}}(1)
\end{aligned}
$$




$$
\begin{aligned}
& \left\|\left|B^{\prime \prime}\right| \operatorname{Tr}_{B^{\prime}}\left(\operatorname{op}^{B^{\prime \prime} \rightarrow B^{\prime}}(\Delta) \cdot\left(U_{2} \cdot \tilde{\varphi}^{B^{\prime \prime} S D}\right)\right)-\varphi^{S D}\right\|_{1} \\
& \leq 20 \cdot 2^{\frac{1}{2} H_{\max }^{\epsilon}(B)_{\varphi}-\frac{1}{2} H_{\min }^{\epsilon}\left(B^{\prime \prime}\right)_{\sigma}}+160 \epsilon \\
& :=\delta_{\mathrm{enc}}(2)
\end{aligned}
$$

\section{Decoding : The Successive Cancellation Step}

This is very similar to the arguments in the proof of Theorem 3.4, and involves two applications of the successive cancellation procedure shown there. The technical details are identical to those described in that proof, so in the interest of brevity, we provide a high level description only. First, Charlie decodes the input of Alice 0 to recover the state $|\eta\rangle^{A_{0} C_{0} R_{0}}$. He keeps this state side, and

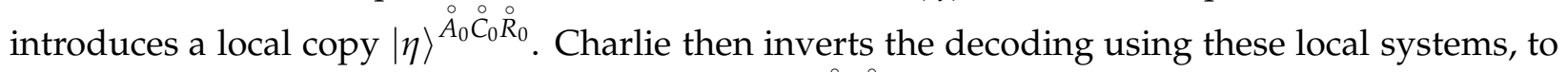
get a state close to GLOBAL 0 , but with the registers $\stackrel{\circ}{C}_{0} \stackrel{\circ}{R}_{0}$ locally available to him.

Charlie then uses the registers $C \stackrel{\circ}{R}_{0} C_{0} D$ to decode Bob's input to recover the state $|\varphi\rangle^{B S D}$. Charlie then keeps Bob's decoded state aside, and introduces the locally prepared state $|\varphi\rangle^{B S} \stackrel{\circ}{ }$. He then inverts the decoding again, using these local systems, to get a state close to GLOBAL 0 , with the systems $\stackrel{\circ}{C}_{0} \stackrel{\circ}{R}_{0} \stackrel{\circ}{S} \stackrel{\circ}{D}$ locally available to him.

In last step of the decoding procedure, Charlie uses the systems $C \stackrel{\circ}{R}_{0} \stackrel{\circ}{C}_{0} \stackrel{\circ}{S}{ }^{\circ} C_{1}$ to decode Alice ${ }_{1}$ s input. This whole procedure outputs a state close to $|\eta\rangle^{R_{0} C_{0} A_{0}} \otimes|\psi\rangle^{R_{1} C_{1} A_{1}} \otimes|\varphi\rangle^{B S D}$ with an error of at most

$$
\delta_{\mathrm{dec}}:=8\left(\sqrt{2 \delta_{\mathrm{dec}}(0)}+\sqrt{2 \delta_{\mathrm{dec}}(1)}+\sqrt{2 \delta_{\mathrm{dec}}(2)}\right)
$$

\section{Encoding}

Firstly, notice that, via Theorem 2.6 in Eq. (enc_Alice),

$$
\begin{aligned}
& \left|A^{\prime \prime}{ }_{0}\right| \operatorname{op}^{A^{\prime \prime}{ }_{0} \rightarrow C_{1} R_{1} A^{\prime}}\left(\omega_{\text {enc }}\left(U_{1}\right)\right) \cdot\left(U_{0} \cdot \tilde{\eta}^{R_{0} A^{\prime \prime}}\right) \\
& =\left|A^{\prime \prime}{ }_{0}\right|\left|A^{\prime \prime}{ }_{1}\right| \mathrm{op}^{A^{\prime \prime}{ }_{0} A^{\prime \prime}{ }_{1} \rightarrow A^{\prime}}\left(\Omega^{\prime}\right)\left(U_{1} \otimes U_{0}\right) \cdot(\tilde{\psi} \otimes \tilde{\eta}) \\
& :=\mathrm{ALICE}^{R_{0} R_{1} C_{1} A^{\prime}}
\end{aligned}
$$

Then, applying a triangle inequality to Eq. (enc_Alice 0 ) and Eq. (enc_Alice, and using Theorem 2.13 and Uhlmann's theorem, one can see that there exists an encoding isometries $V_{\mathrm{ENC} \_ \text {ALICE }}^{A_{0} A_{1} \rightarrow A^{\prime}}$ and $V_{\text {ENC_BOB }}^{B \rightarrow B^{\prime}}$ such that

$$
\begin{aligned}
& \left\|c_{1} \cdot \operatorname{ALICE}^{R_{0} R_{1} C_{1} A^{\prime}}-V_{\text {ENC_ALICE }} \cdot\left(\psi^{R_{1} C_{1} A_{1}} \otimes \eta^{R_{0} A_{0}}\right)\right\|_{1} \leq 2 \sqrt{2 \delta_{\text {enc }}(0)+2 \delta_{\text {enc }}(1)} \\
& \left\|c_{2} \cdot\left|B^{\prime \prime}\right| \operatorname{op}^{B^{\prime \prime} \rightarrow B^{\prime}}(\Delta) \cdot\left(U_{2} \cdot \tilde{\varphi}^{B^{\prime \prime} D S}\right)-V_{\text {ENC_BOB }} \cdot \varphi^{B D S}\right\|_{1} \leq 2 \sqrt{2 \delta_{\text {enc }}(2)}
\end{aligned}
$$

where $c_{1}$ and $c_{2}$ are appropriate normalisation factors.

$$
\begin{aligned}
& \operatorname{ALICE}^{R_{0} R_{1} C_{1} A^{\prime}} \otimes\left|B^{\prime \prime}\right| \mathrm{op}^{B^{\prime \prime} \rightarrow B^{\prime}}(\Delta) \cdot\left(U_{2} \cdot \tilde{\varphi}^{B^{\prime \prime} D S}\right) \\
& =\mathrm{GLOBAL}_{0}
\end{aligned}
$$


Then, by some standard algebraic manipulation we see that

$$
\begin{aligned}
& \left\|c_{1} c_{2} \cdot \mathrm{GLOBAL}_{0}-\left(V_{\mathrm{ENC} \_\mathrm{ALICE}} \otimes V_{\mathrm{ENC} \_ \text {воB }}\right) \cdot\left(\eta^{R_{0} A_{0}} \psi^{R_{1} C_{1} A_{1}} \varphi^{S D B}\right)\right\|_{1} \\
& \leq\left(1+2 \sqrt{2 \delta_{\text {enc }}(2)}\right) \times\left(2 \sqrt{2 \delta_{\text {enc }}(0)+2 \delta_{\text {enc }}(1)}\right)+2 \sqrt{2 \delta_{\text {enc }}(2)} \\
& :=\delta_{\text {enc }}
\end{aligned}
$$

We can now concude the proof by the usual arguments.

\section{The Interference Channel}

In this section we prove inner bounds for partially entanglement assisted entanglement transmission through the Quantum Interference Channel (QIC) $\mathcal{N}^{A^{\prime} B^{\prime} \rightarrow C D}$. We wish for Alice to send EPR pairs to Charlie and for Bob to send EPR pairs to Damru. Note that, for a fixed control state $|\sigma\rangle^{A^{\prime \prime} A^{\prime} B^{\prime \prime} B^{\prime}}:=|\Omega\rangle^{A^{\prime \prime} A^{\prime}}|\Delta\rangle^{B^{\prime \prime} B^{\prime}}$, one can consider this situation as two point to point channels, one from Alice to Charlie and one from Bob to Damru. In that case, the achievable region becomes a rectangle of all non negative rate pairs less than $\left.\left(I_{\min }^{\epsilon}\left(A^{\prime \prime}>C\right)_{\sigma}, I_{\min }^{\epsilon}\left(B^{\prime \prime}>D\right)\right)_{\sigma}\right)$ (suppressing the additive $\log$ terms).

In order to show that a larger region is achievable, we use splitting schemes and successive cancellation. Essentially, we split Alice into two senders, Alice ${ }_{1}$ and Alice, ${ }_{2}$, and we require Alice ${ }_{1}$ 's input to be decoded by Damru instead of Charlie. This allows Damru to treat Alice ${ }_{1}$ 's input as side information while decoding Bob's input, which allows us to boost Bob's rate. Alice's rate to Charlie however, takes a hit because of this. Using a splitting scheme to do this allows us to adjust the amount of resources that Alice dedicates towards boosting Bob's rate, with the extreme cases $\theta \in\{0,1\}$ corresponding to situations when either Alice does not help bob at all (the case of the two point to point channels) ot when Alice dedicates all her resources to help Bob while her own rates drops to 0 .

The precise statements can be found in Theorem 5.1 and Theorem 5.2.

Proposition 5.1 Consider the quantum interference channel $\mathcal{N}^{A^{\prime} B^{\prime} \rightarrow C D}$. Consider a pure 'control state' $|\sigma\rangle^{A^{\prime \prime} B^{\prime \prime} A^{\prime} B^{\prime}}:=|\Omega\rangle^{A^{\prime \prime} A^{\prime}}|\Delta\rangle^{B^{\prime \prime} B^{\prime}}$. Let $|\psi\rangle^{A_{1} C_{1} R_{1}}$ and $|\eta\rangle^{A_{0} R_{0}}$ be the states that are to be sent by Alice to Charlie and Damru respectively and let $|\phi\rangle^{B D_{0} S}$ be the state to be sent from Bob to Damru, where $C_{1}$, $D_{0}$ model the side information about the respective messages $A_{1}, B$ that Charlie and Damru possess and $R_{0}, R_{1}, S$ are reference systems that are untouched by channel and coding operators. Let $\mathbb{I}$ denote the identity superoperator. For $\theta \in[0,1]$, let $U_{\theta}^{A^{\prime \prime}}$ be a splitting scheme. We define $|\sigma(\theta)\rangle^{A_{0}^{\prime \prime} A_{1}^{\prime \prime} A^{\prime} B^{\prime \prime} B^{\prime}}:=$ $U_{\theta}|\Omega\rangle^{A^{\prime \prime} A^{\prime}}|\Delta\rangle^{B^{\prime \prime} B^{\prime}}$ and

$$
\sigma(\theta)^{A_{0}^{\prime \prime} A_{1}^{\prime \prime} B^{\prime \prime} C D}:=\left(\mathcal{N}^{A^{\prime} B^{\prime} \rightarrow C D} \otimes \mathbb{I}^{A_{0}^{\prime \prime} A_{1}^{\prime \prime} B^{\prime \prime}}\right)\left(\sigma(\theta)^{A_{0}^{\prime \prime} A_{1}^{\prime \prime} A^{\prime} B^{\prime \prime} B^{\prime}}\right) .
$$

Then there exist encoding maps $\mathcal{A}^{A_{0} A_{1} \rightarrow A^{\prime}}$ and $\mathcal{B}^{B \rightarrow B^{\prime}}$ and decoding maps $\mathcal{C}^{C C_{1} \rightarrow A_{1} C_{1}}$ and $\mathcal{D}^{D D_{0} \rightarrow A_{0} B D_{0}}$ such that

$$
\|(\mathcal{C} \otimes \mathcal{D}) \circ \mathcal{N} \circ(\mathcal{A} \otimes \mathcal{B}) \cdot(\psi \otimes \eta \otimes \varphi)-\psi \otimes \eta \otimes \varphi\|_{1} \leq \delta
$$


Here, $\delta=\delta_{\mathrm{enc}}+\delta_{\mathrm{dec}}$ where,

$$
\begin{aligned}
& \delta_{\mathrm{enc}}=\left(1+2 \sqrt{2 \delta_{\mathrm{enc}}(2)}\right) \times\left(2 \sqrt{2 \delta_{\mathrm{enc}}(0)+2 \delta_{\mathrm{enc}}(1)}\right)+2 \sqrt{2 \delta_{\mathrm{enc}}(2)} \\
& \delta_{\mathrm{dec}}=8 \sqrt{2 \delta_{\mathrm{dec}}(0)}+4 \sqrt{2 \delta_{\mathrm{dec}}(1)}+2 \sqrt{2 \delta_{\mathrm{dec}}(2)}
\end{aligned}
$$

and

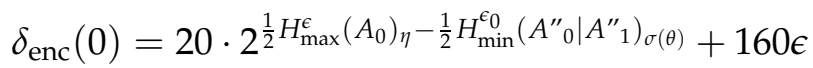

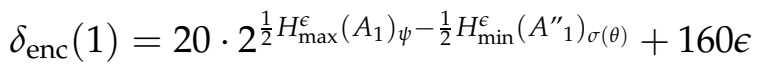

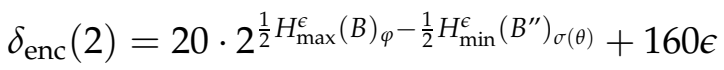

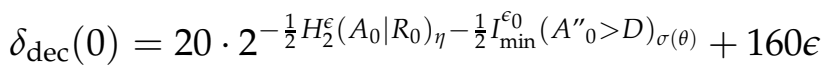

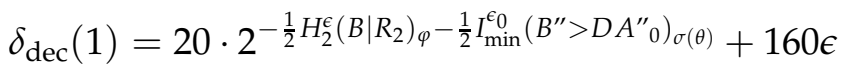

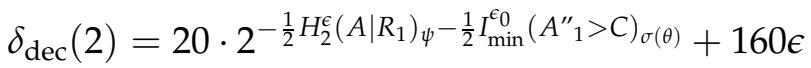

where $\epsilon_{0}=\frac{\epsilon^{2}}{800}$.

We are now ready to state our main one shot coding theorem. In this case, we denote by $Q_{0}$ the number of qubits available to Alice for sending to Damru, to use as side information to boost Bob's rate. The quantities of interest however are $\left(Q_{A}, E_{A}, Q_{B}, E_{B}\right)$ which denote, in order, the number of message qubits and ebits available to Alice, and the analogous quantities for Bob .

Theorem 5.2 Consider the setting of Theorem 5.1 Let $Q_{A}, E_{A}, Q_{B}, E_{B}$ be the number of message qubits and number of available ebits of Alice and Bob respectively. Additionally, let $Q_{0}$ denote the number message qubits available to Alice for transmission to Damru. Let $\theta, \epsilon \in[0,1]$ and $\epsilon_{0}:=\frac{\epsilon^{2}}{800}$. Then there exist encoding and decoding maps such that any message cum ebit rate 4-tuple satisfying the following inequalities, is achievable with error at most $280 \sqrt{\epsilon}$ achievable for partial entanglement assisted entanglement transmission

$$
\begin{aligned}
& Q_{0}<I_{\min }^{\epsilon_{0}}\left(A^{\prime \prime}{ }_{0}>D\right)_{\sigma(\theta)}+\log \epsilon^{2} \\
& Q_{0}<H_{\min }^{\epsilon_{0}}\left(A^{\prime \prime}{ }_{0} \mid A^{\prime \prime}{ }_{1}\right)_{\sigma(\theta)}+\log \epsilon^{2} \\
& Q_{A}+E_{A}<H_{\min }^{\epsilon}\left(A^{\prime \prime}{ }_{1}\right)_{\sigma(\theta)}+\log \epsilon^{2} \\
& Q_{A}-E_{A}<I_{\min }^{\epsilon_{0}}\left(A^{\prime \prime}{ }_{1}>C\right)_{\sigma(\theta)}+\log \epsilon^{2} \\
& Q_{B}+E_{B}<H_{\min }^{\epsilon}\left(B^{\prime \prime}\right)_{\sigma(\theta)}+\log \epsilon^{2} \\
& Q_{B}-E_{B}<I_{\min }^{\epsilon_{0}}\left(B^{\prime \prime}>A^{\prime \prime}{ }_{0} D\right)_{\sigma(\theta)}+\log \epsilon^{2}
\end{aligned}
$$

The proofs of Theorem 5.1 and Theorem 5.2 are essentially the same as their counterparts for the QMAC, with some technical changes due to the presence of two decoders. These changes can be dealt with easily via standard techniques, and hence we omit these proofs.

Remark 5.3 In Section 6, when we state the asymptotic iid extension of this theorem, we will fix a value of $Q_{0}$ and not mention it explicitly to highlight the region of interest. 


\section{Extension to the Asymptotic IID Regime}

The bounds proved in Theorem 4.2 and Theorem 5.2 can be easily extended to the case when many uses of the channel are allowed. We show in this section that one can extend Theorem 4.2 to the asymptotic iid regime to recover the best known inner bounds for entanglement transmission over the QMAC in that regime [YDH05]. Similar arguments allow us to extend Theorem 5.2 to that regime and prove the first non trivial asymptotic iid inner bounds for entanglement transmission over the QIC.

The idea is simple and uses the quantum asymptotic equipartition theorem (QAEP) Theorem 2.4 As an example, consider that we are given the QMAC $\mathcal{N}^{A^{\prime} B^{\dagger} \rightarrow C}$ and the control state $|\sigma\rangle$ as usual, but now we are allowed $n \in \mathbb{N}$ uses of the channel along with $n$ tensored copies of the control state. Suppose that $\delta>0$ is some small positive number and fix a $\theta \in[0,1]$. Define $|\sigma\rangle$ as usual with respect to this fixed $\theta$. Then, for some large enough $n$, using the Theorem 2.4, and repeating the proof of Theorem 4.2, we see that the achievable region, for the fixed $\theta$ for partial entanglement assisted entanglement transmission is given by

$$
\begin{aligned}
& n\left(Q_{A}+E_{A}\right) \leq n H\left(A^{\prime \prime}{ }_{1}\right)_{\sigma}+n H\left(A^{\prime \prime}{ }_{0} \mid A^{\prime \prime}{ }_{1}\right)_{\sigma}-n \delta \\
& n\left(Q_{A}-E_{A}\right) \leq n I\left(A^{\prime \prime}{ }_{0}>C\right)_{\mathcal{U}_{\mathcal{N}} \cdot \sigma}+n I\left(A^{\prime \prime}{ }_{1}>A^{\prime \prime}{ }_{0} B^{\prime \prime} C\right) \mathcal{U}_{\mathcal{N} \cdot \sigma}-n \delta \\
& n\left(Q_{B}+E_{B}\right) \leq n H\left(B^{\prime \prime}\right)_{\sigma}-n \delta \\
& n\left(Q_{B}-E_{B}\right) \leq n I\left(B^{\prime \prime}>A^{\prime \prime}{ }_{0} C\right)_{\mathcal{U}_{\mathcal{N}} \cdot \sigma}-n \delta
\end{aligned}
$$

The usual continuity arguments with respect to $\theta \in[0,1]$ along with the invariance of the Shannon entropy and the coherent information under isometries give the full pentagonal region:

$$
\begin{aligned}
& Q_{A}+E_{A} \leq H\left(A^{\prime \prime}\right)_{\sigma}-\delta \\
& Q_{A}-E_{A} \leq I\left(A^{\prime \prime}>B^{\prime \prime} C\right)_{\mathcal{U}_{\mathcal{N}} \cdot \sigma}-\delta \\
& Q_{B}+E_{B} \leq H\left(B^{\prime \prime}\right)_{\sigma}-\delta \\
& Q_{B}-E_{B} \leq I\left(B^{\prime \prime}>A^{\prime \prime} C\right) \mathcal{U}_{\mathcal{N}} \cdot \sigma-\delta \\
& Q_{A}-E_{A}+Q_{B}-E_{B}<I\left(A^{\prime \prime} B^{\prime \prime}>C\right)_{\mathcal{U}_{\mathcal{N}} \cdot \sigma}-2 \delta
\end{aligned}
$$

Note that setting $E_{A}$ and $E_{B}$ to 0 , we recover the bounds shown in [YDH05]. We state the above arguments as a theorem:

Theorem 6.1 Given a quantum multiple access channel $\mathcal{N}^{A^{\prime} B^{\prime} \rightarrow C}$ all rate points in the closure of the following region are achievable for partial entanglement assisted entanglement generation :

$$
\bigcup_{k=1}^{\infty} \frac{1}{k} \mathcal{Q}\left(\mathcal{N}^{\otimes k}\right)
$$


where $\mathcal{Q}\left(\mathcal{N}^{\otimes k}\right)$ is the set of non negative rate tuples $\left(Q_{A}, E_{A}, Q_{B}, E_{B}\right)$ in the set

$$
\begin{aligned}
Q_{A}+E_{A}<H\left(A^{\prime \prime k}\right)_{\sigma_{k}} \\
Q_{A}-E_{A}<I\left(A^{\prime \prime k}>B^{\prime \prime k} C^{k}\right) \mathcal{U}_{\mathcal{N}} \cdot \sigma_{k} \\
Q_{B}+E_{B}<H\left(B^{\prime \prime k}\right)_{\sigma_{k}} \\
Q_{B}-E_{B}<I\left(B^{\prime \prime k}>A^{\prime \prime k} C^{k}\right) \mathcal{U}_{\mathcal{N}} \cdot \sigma_{k} \\
Q_{A}-E_{A}+Q_{B}-E_{B}<I\left(A^{\prime \prime k} B^{\prime \prime k}>C^{k}\right) \mathcal{U}_{\mathcal{N}} \cdot \sigma_{k}
\end{aligned}
$$

where $\left|\sigma_{k}\right\rangle^{A^{\prime \prime k} B^{\prime \prime} A^{\prime} B^{\prime} k}:=|\Omega\rangle^{A^{\prime \prime} A^{\prime k}}|\Delta\rangle^{B^{\prime \prime} B^{\prime k}}$.

Before stating the analogous theorem for the QIC, we note that, the set of achievable points for the QIC is actually larger than the one shown in Theorem 5.2. This is via the simple observation that, we only allowed Alice to help Bob by taking a hit to her own rate. We would get a different set of achievable points if we allow Bob to help Alice. The union of these two regions is the complete rate region.

Theorem 6.2 Given a quantum interference channel $\mathcal{N} \otimes k$, the control state $|\sigma\rangle^{A^{\prime \prime} A^{\prime} B^{\prime \prime} B^{\prime}}$ the following regularised rate region is achievable for partial entanglement assisted entanglement transmission :

$$
\bigcup_{k=1}^{\infty} \frac{1}{k} \mathcal{Q}\left(\mathcal{N}^{\otimes k}\right)
$$

For each $k \in \mathbb{N}$,

$$
\mathcal{Q}\left(\mathcal{N}^{\otimes k}\right)=\bigcup \mathcal{A}_{\theta}^{k} \bigcup \mathcal{B}_{\theta}^{k}
$$

where, for a fixed $\theta \in[0,1], \mathcal{A}_{\theta}^{k}$ is the set of all non-negative tuples $\left(Q_{A}, E_{A}, Q_{B}, E_{B}\right)$ such that

$$
\begin{aligned}
& Q_{A}+E_{A}<H\left(A_{1}^{\prime \prime k}\right)_{\sigma_{k}(\theta)} \\
& Q_{A}-E_{A}<I\left(A_{1}^{\prime \prime k}>C_{1}^{k}\right) \mathcal{U}_{\mathcal{N} \cdot \sigma_{k}(\theta)} \\
& Q_{B}+E_{B}<H\left(B^{\prime \prime k}\right)_{\sigma_{k}(\theta)} \\
& Q_{B}-E_{B}<I\left(B^{\prime \prime k}>A_{0}^{\prime \prime k} C_{2}^{k}\right)_{\mathcal{U}_{\mathcal{N}} \cdot \sigma_{k}(\theta)}
\end{aligned}
$$

where $\left|\sigma_{k}\right\rangle^{A^{\prime \prime k} B^{\prime \prime} A^{\prime k} B^{\prime k}}:=|\Omega\rangle^{A^{\prime \prime k} A^{\prime k}}|\Delta\rangle^{B^{\prime \prime} B^{\prime k}}$ and $\left|\sigma_{k}\right\rangle:=U^{A^{\prime \prime k} \rightarrow A_{0}^{\prime \prime k} A_{1}^{\prime \prime k}}\left|\sigma_{k}\right\rangle$. We assume that $U_{\theta}$ is a splitting scheme. Analogously, $\mathcal{B}_{\theta}^{k}$ is the set of those points which are obtained when the splitting isometry acts on the system $B^{\prime \prime}$.

\section{References}

[Ahl71] R. Ahlswede. Multi-way communication channels. In Proceedings of 2nd International Symposium on Information Theory (ISIT), pages 23-52, 1971.

[BD10] F. Buscemi and N. Datta. The quantum capacity of channels with arbitrarily correlated noise. IEEE Transactions on Information Theory, 56(3):1447-1460, 2010. 
[CMGE08] Chong, H., Motani, M., Garg, H., and El Gamal, H. On the Han-Kobayashi region for the interference channel. IEEE Transactions on Information Theory, 54:3188-3195, 2008.

[CNS21] Chakraborty, S., Nema, A., and Sen, P. A multi sender decoupling theorem and simultaneous decoding for the quantum mac. Available at arXiv:2101.1000, 2021.

[DBWR14] Frédéric Dupuis, Mario Berta, Jürg Wullschleger, and Renato Renner. One-shot decoupling. Communications in Mathematical Physics, 328(1):251-284, May 2014.

[Dev05] I. Devetak. The private classical capacity and quantum capacity of a quantum channel. IEEE Transactions on Information Theory, 51(1):44-55, 2005.

[Dup10] Frédéric Dupuis. The decoupling approach to quantum information theory. PhD thesis, Université de Montréal, 2010.

[FHS ${ }^{+}$12] O. Fawzi, P. Hayden, I. Savov, P. Sen, and M. Wilde. Classical communication over a quantum interference channel. IEEE Transactions on Information Theory, 58:3670-3691, 2012.

[GRUW01] Alexander Grant, Bixio Rimoldi, Rüdiger Urbanke, and Philip Whiting. Rate-splitting multiple access for discrete memoryless channels. Information Theory, IEEE Transactions on, 47:873 - 890, 042001.

[HDW08] M. Hsieh, I. Devetak, and A. Winter. Entanglement-assisted capacity of quantum multiple-access channels. IEEE Transactions on Information Theory, 54(7):3078-3090, 2008.

[HHYW07] Patrick Hayden, Michal Horodecki, Jon Yard, and Andreas Winter. A decoupling approach to the quantum capacity. Open Systems E Information Dynamics, 15, 032007.

[HK81] Han, T. and Kobayashi, K. A new achievable rate region for the interference channel. IEEE Transactions on Information Theory, 27:49-60, 1981.

[KW03] Dennis Kretschmann and Reinhard Werner. Tema con variazioni: Quantum channel capacity. New Journal of Physics, 6, 112003.

[Lia72] H. Liao. Multiple access channels. PhD thesis, University of Hawai, 1972.

[Llo97] Seth Lloyd. Capacity of the noisy quantum channel. Phys. Rev. A, 55:1613-1622, Mar 1997.

[SDTR13] Oleg Szehr, Frédéric Dupuis, Marco Tomamichel, and Renato Renner. Decoupling with unitary approximate two-designs. New Journal of Physics, 15(5):053022, may 2013.

[Sen12] P. Sen. Achieving the Han-Kobayashi inner bound for the quantum interference channel. In IEEE International Symposium on Information Theory (ISIT), pages 736-740, 2012. Full version at arXiv:1109.0802.

[Sen18a] Pranab Sen. A one-shot quantum joint typicality lemma. arXiv e-prints, page arXiv:1806.07278, June 2018. 
[Sen18b] Pranab Sen. Inner bounds via simultaneous decoding in quantum network information theory. arXiv e-prints, page arXiv:1806.07276, June 2018.

[Sho02] Peter Shor. The quantum channel capacity and coherent information. Lecture Notes, MSRI workshop on quantum computation, 2002.

[TCR09] M. Tomamichel, R. Colbeck, and R. Renner. A fully quantum asymptotic equipartition property. IEEE Transactions on Information Theory, 55(12):5840-5847, 2009.

[TCR10] M. Tomamichel, R. Colbeck, and R. Renner. Duality between smooth min- and maxentropies. IEEE Transactions on Information Theory, 56(9):4674-4681, 2010.

[VDTR13] A. Vitanov, F. Dupuis, M. Tomamichel, and R. Renner. Chain rules for smooth minand max-entropies. IEEE Transactions on Information Theory, 59(5):2603-2612, 2013.

[Win01] Andreas Winter. The capacity of the quantum multiple-access channel. Information Theory, IEEE Transactions on, 47:3059 - 3065, 122001.

[YDH05] J. Yard, I. Devetak, and P. Hayden. Capacity theorems for quantum multiple access channels. In Proceedings. International Symposium on Information Theory, 2005. ISIT 2005., pages 884-888, 2005.

\section{A Proofs of Important Lemmas}

Lemma A.1 Given $\theta, \theta^{\prime} \in[0,1]$ such that $\left|\theta-\theta^{\prime}\right| \leq \delta$, we have that

$$
P\left(\Omega^{\prime}(\theta)^{A^{\prime \prime}{ }_{0}{ }{ }^{\prime \prime}{ }_{1} B E}, \Omega^{\prime}\left(\theta^{\prime}\right) A^{{ }^{\prime \prime}{ }_{0} A^{\prime \prime}{ }_{1} B E}\right) \leq O(\sqrt{\delta})
$$

Proof:[Proof of Theorem A.1] For the course of the proof we will neglect to mention the registers in the superscript to ease the notation, unless necessary. Since both $\Omega^{\prime}(\theta)$ and $\Omega^{\prime}\left(\theta^{\prime}\right)$ are pure, we will use the identity :

$$
P\left(\Omega^{\prime}(\theta), \Omega^{\prime}\left(\theta^{\prime}\right)\right)=\sqrt{1-\left|\left\langle\Omega^{\prime}(\theta) \mid \Omega^{\prime}\left(\theta^{\prime}\right)\right\rangle\right|^{2}}
$$

Recall that since

$$
\left|\Omega^{\prime}\right\rangle^{A^{\prime \prime}{ }_{0} A_{0}{ }_{0} B E}(\theta)=U_{\mathcal{N}}^{A^{\prime}} \circ U_{f}^{A^{\prime \prime}{ }_{0} A^{\prime \prime}{ }_{1} A^{\prime}}\left(\sum_{u \in \mathcal{A}} \sqrt{P_{U}^{\theta}(u)}|u\rangle^{A^{\prime \prime}{ }_{0}}\right) \otimes\left(\sum_{v \in \mathcal{A}} \sqrt{P_{V}^{\theta}(v)}|v\rangle^{A^{\prime \prime}{ }_{0}}\right)|0\rangle^{A^{\prime}}
$$

and similarly for $\left|\Omega^{\prime}\right\rangle\left(\theta^{\prime}\right)$,

$$
\left\langle\Omega^{\prime}(\theta) \mid \Omega^{\prime}\left(\theta^{\prime}\right)\right\rangle=F\left(P_{U}^{\theta}, P_{U}^{\theta^{\prime}}\right) F\left(P_{V}^{\theta}, P_{V}^{\theta^{\prime}}\right)
$$

It is thus sufficient to show that the distributions $P_{U}^{\theta}$ and $P_{V}^{\theta}$ are close to $P_{U}^{\theta^{\prime}}$ and $P_{V}^{\theta^{\prime}}$ respectively. Then, recalling the explicit form of $P_{U}^{\theta}$ observe that:

$$
\begin{aligned}
\left\|P_{U}^{\theta^{\prime}}-P_{U}^{\theta}\right\|_{1} & =\left|\left(1-\theta+\theta P_{A}(0)\right)-\left(1-\theta^{\prime}+\theta^{\prime} P_{A}(0)\right)\right|+\sum_{i \neq 0}\left|\theta P_{A}(i)-\theta^{\prime} P_{A}(i)\right| \\
& \leq\left|\theta-\theta^{\prime}\right|+\left|\theta-\theta^{\prime}\right| \sum_{i \in \mathcal{A}} P_{A}(i) \\
& \leq 2 \delta
\end{aligned}
$$


Next, observe that, for any $i \in \mathcal{A}$

$$
P_{V}^{\theta}(i)=\frac{F_{A}(i)}{F_{U}^{\theta}(i)}-\frac{F_{A}(i-1)}{F_{U}^{\theta}(i-1)}
$$

It holds that

$$
\begin{aligned}
F_{A}(i) F_{U}^{\theta}(i-1)-F_{A}(i-1) F_{U}^{\theta}(i) & =\left(P_{A}(i)+F_{A}(i-1)\right) F_{U}^{\theta}(i-1)-F_{A}(i-1)\left(\theta F_{A}(i)+1-\theta\right) \\
& =\left(P_{A}(i)+F_{A}(i-1)\right) F_{U}^{\theta}(i-1)-F_{A}(i-1)\left(F_{U}^{\theta}(i-1)+\theta P_{A}(i)\right) \\
& =P_{A}(i)\left(\theta F_{A}(i-1)+1-\theta\right)-\theta F_{A}(i-1) P_{A}(i) \\
& =(1-\theta) P_{A}(i)
\end{aligned}
$$

Denote $F_{U}^{\theta}(i) F_{U}^{\theta}(i-1):=g(\theta)$. Then,

$$
\begin{aligned}
& \left|g(\theta)-g\left(\theta^{\prime}\right)\right| \\
& =\left|F_{U}^{\theta}(i) F_{U}^{\theta}(i-1)-F_{U}^{\theta^{\prime}}(i) F_{U}^{\theta^{\prime}}(i-1)\right| \\
& \leq\left|F_{U}^{\theta}(i) F_{U}^{\theta}(i-1)-F_{U}^{\theta^{\prime}}(i) F_{U}^{\theta}(i-1)\right|+\left|F_{U}^{\theta^{\prime}}(i) F_{U}^{\theta}(i-1)-F_{U}^{\theta^{\prime}}(i) F_{U}^{\theta^{\prime}}(i-1)\right| \\
& \leq 4 \delta
\end{aligned}
$$

Let $p^{*}=\min _{i \in \mathcal{A}} P_{A}(i)$. Then,

$$
\begin{aligned}
g(\theta) & \geq\left(1-\theta+\theta p^{*}\right)^{2} \\
\geq p^{* 2} & \left|\frac{1-\theta}{g(\theta)}-\frac{1-\theta^{\prime}}{g\left(\theta^{\prime}\right)}\right| \\
\left|P_{V}^{\theta}(i)-P_{V}^{\theta^{\prime}}(i)\right| & =P_{A}(i) \\
& =\frac{P_{A}(i)}{\left|g(\theta) g\left(\theta^{\prime}\right)\right|} \cdot\left|(1-\theta) g\left(\theta^{\prime}\right)-\left(1-\theta^{\prime}\right) g(\theta)\right| \\
& \stackrel{(a)}{\leq} \frac{P_{A}(i)}{p^{* 4}} \cdot c \cdot \delta
\end{aligned}
$$

where $c$ is some constant and we have used the triangle inequality and the lower bound for $g(\theta)$ in $(a)$.

Then, the above bound implies that:

$$
\left\|P_{V}^{\theta}-P_{V}^{\theta^{\prime}}\right\|_{1} \leq O(\delta)
$$

Using the property that $F(P, Q) \geq 1-\|P-Q\|_{1}$ for any two distributions $P$ and $Q$, we see that

$$
\left|\left\langle\Omega^{\prime}(\theta) \mid \Omega^{\prime}\left(\theta^{\prime}\right)\right\rangle\right|^{2} \geq 1-O(\delta)
$$

This concludes the proof. 